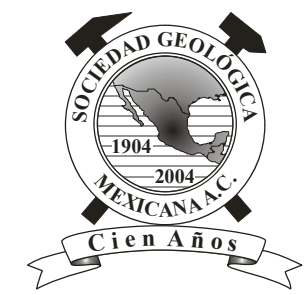

\title{
Retos y oportunidades para el aprovechamiento y manejo ambiental del ex lago de Texcoco
}

\author{
Arón Jazcilevich Diamant ${ }^{1}$, Christina Siebe ${ }^{2, *}$, Claudio Estrada ${ }^{3}$, Javier Aguillón ${ }^{4}$, Alberto \\ Rojas $^{5}$, Elizabeth Chávez García ${ }^{1}$, Claudia Sheinbaum Pardo ${ }^{4}$ \\ ${ }^{1}$ Centro de Ciencias de la Atmósfera, Universidad Nacional Autónoma de México, Ciudad Universitaria, México D.F. CP 04510, \\ México. \\ ${ }^{2}$ Instituto de Geología, Universidad Nacional Autónoma de México, Ciudad Universitaria, México D.F. CP 04510, México. \\ ${ }^{3}$ Instituto de Energías Renovables, Universidad Nacional Autónoma de México, Temixco, Mor. CP 62580, México. \\ ${ }^{4}$ Instituto de Ingeniería, Universidad Nacional Autónoma de México, Ciudad Universitaria, México D.F. CP 04510, México. \\ ${ }^{5}$ Consultora Geoda, Geosistemas y Desarrollo Ambiental S.C., Avenida Insurgentes Sur No. 3493 Edif. 19, Depto. 102, Col. Villa \\ Olímpica, Deleg. Tlalpan, México D.F. CP 14020, México. \\ *siebe@unam.mx
}

\section{Resumen}

La Zona Federal del ex lago de Texcoco es una amplia superficie libre de construcciones inmersa en una gran metrópoli. Tiene importantes potenciales de uso, tanto para el desarrollo de proyectos ecológicos y recreativos como para la generación de energías renovables; además cumple funciones de regulación hidrológica, térmica y de calidad del aire. En este trabajo se presentan cuatro propuestas para el manejo del ex lago de Texcoco en favor del desarrollo económico y ambiental de la Zona Metropolitana del Valle de México a largo plazo. Éstas son: a) rehabilitación de suelos y vegetación, b) regeneración de zonas lacustres, c) manejo de residuos sólidos orgánicos y d) generación de electricidad y calor con energía solar. Estas propuestas surgieron a partir de la opinión de expertos, durante la realización de dos talleres multidisciplinarios. Las propuestas son compatibles entre sí, tienen cauce legal y aprovechan la circunstancia geográfica y ambiental del ex lago de Texcoco de forma sostenible y productiva. Se concluye que una combinación de las diversas opciones de uso aquí descritas potenciará las funciones del suelo de manera integral y permitirá mitigar los problemas ambientales y de gestión hidráulica que afectan a esta región.

Recientemente se ha anunciado la ampliación del aeropuerto de la Ciudad de México. Esto pone en una encrucijada la utilización de la Zona Federal del ex lago de Texcoco: se le convierte en un aeropuerto o se le aprovecha para mitigar problemas de energía, gestión hidráulica y de calidad del aire para promover el desarrollo ambiental, económico y social de la región central del país, como pretenden las propuestas aquí descritas.

Palabras clave: Calidad del aire, revegetación de suelos salino-sódicos, regulación hidrológica, energía renovable, manejo de residuos, aeropuerto.

\begin{abstract}
The lacustrian plain of former lake Texcoco is an extensive non-urbanized area within a large megacity. It has high land use potential for the development of conservation and recreation projects, and also for the generation of renewable energy. Besides, it has the potential of fulfilling relevant ecological functions such as the regulation of surface run-off, temperature and air quality. Four proposals are presented for the management of this area to promote the economic development of the central region of Mexico under sound environmental conditions. These proposals are: a) regeneration of soil and vegetation, b) regeneration of lacustrine areas, c) management of solid organic residues, and d) generation of solar electricity and heat. These proposals arose from expert opinion during
\end{abstract}


two multidisciplinary workshops. The proposals are compatible, have legal foundations and take advantage of the geography and current environment of the Texcoco area in a sustainable and productive way. We conclude that a combination of the options presented increases land use potential and allows mitigation of the various environmental and water management problems affecting this region.

Recently, the expansion of the International Airport of Mexico City in the Texcoco area was announced. This puts the development of this area at a crossroads: it could be used for an airport or it could be used to mitigate energy, hydraulic and air quality problems to promote the environmental, economic and social development of the central region of the country, as the described proposals claim.

Keywords: Air quality, revegetation of saline and alkaline soil, hydrological regulation, renewable energy, urban waste management, airport.

\section{Introducción}

1.1. Texcoco el futuro de la Zona Metropolitana del Valle de México

Con 20 millones de habitantes, la Zona Metropolitana del Valle de México (ZMVM) incluye la capital política del país y 59 municipios del Estado de México. Esta área representa el centro cultural, financiero y el motor más importante de la actividad económica a nivel nacional. La ZMVM forma parte de una megalópolis con aproximadamente el $20 \%$ de la población de México y en donde se produce casi el $35 \%$ del PIB nacional (Escobar y Jiménez, 2009; INEGI, 2011a, 2011b). El PIB por persona en el Distrito Federal es de US\$ 20400 ubicándose en Latinoamérica detrás de Buenos Aires y São Paolo (Siemens, 2010). Si bien la ZMVM sigue siendo un centro industrial importante, en los últimos 15 años los servicios dominan cada vez más.

Promover el desarrollo económico de esta región resulta estratégico para el país y, para que éste sea sustentable y sostenible deben tomarse decisiones que aborden sus problemas en forma efectiva. En la actualidad esta problemática incluye diversos problemas ambientales como la mitigación de los efectos del cambio climático, su relación con la generación y uso eficiente de la energía, aunados a los derivados por la presencia de grandes asentamientos humanos y su actividad económica en una cuenca hidrológica semiárida y endorreica. Por esta razón los aspectos ambientales que se abordan en el presente documento son: uso y consumo de energía, manejo de residuos sólidos orgánicos urbanos, gestión hidráulica y de la calidad del aire.

En grandes aglomeraciones urbanas se destina una gran porción del territorio al soporte de infraestructura. En la cuenca de México, las zonas urbanizadas ocupan actualmente más del $70 \%$ de los terrenos de las partes bajas, es decir, de la antigua planicie lacustre y de los piedemontes. El sellamiento de la superficie limita importantes funciones ecológicas del suelo, como su capacidad de soportar una cobertura vegetal con fines recreativos y de producción de alimentos, así como la regulación de balance hídrico y la recarga de los acuíferos, la regulación térmica y de la calidad del aire (Kopp et al., 1982; Stasch, 1996). Todo lo anterior afecta directa o indirectamente el bienestar de los habitantes de la metrópoli.

La Zona Federal del ex lago de Texcoco (ZFLT) conforma una gran superficie $\left(60 \mathrm{~km}^{2}\right)$ en las partes bajas de la cuenca que aún no ha sido afectada por el crecimiento urbano. Por consiguiente tiene un papel central en la preservación y viabilidad de la megalópolis. Sin embargo, el crecimiento poblacional ha seguido ejerciendo una gran presión sobre esta superficie, priorizando sus potenciales de soporte de infraestructura urbana, como lo es la ampliación del aeropuerto de la ciudad, o los de depósito de desechos sólidos urbanos en el Bordo Poniente. Desde la desecación casi total del lago, estos terrenos con suelos salino-sódicos carecen de una cobertura vegetal en una gran porción de su superficie. Lo anterior propicia la erosión eólica y la consiguiente emisión de partículas a la atmósfera en detrimento de la calidad del aire de la metrópoli y la salud de sus habitantes.

La decisión sobre el aprovechamiento de esta gran superficie requiere por lo tanto de un ejercicio de planeación urbana, de analizar opciones que pudieran reducir la emisión de aeropartículas y de aprovechar otros potenciales de uso considerando las oportunidades que brindan las nuevas tecnologías con una visión de largo plazo.

El objetivo del presente trabajo fue generar propuestas conceptuales para el uso de suelo en la ZFLT que mitiguen los problemas de gestión hidráulica, generación de energía renovable, manejo de residuos y calidad del aire para el desarrollo sustentable de la ZMVM. Con base en información disponible y los resultados de dos talleres con expertos se generaron propuestas para el manejo de la ZFLT en favor del desarrollo económico y ambiental de la ZMVM. El eje de estas propuestas gira en torno a que la ZFLT sea una oportunidad para contribuir a resolver integralmente la problemática de la región. Además, las propuestas pueden combinarse para que una potencie a la otra y están concebidas en el contexto de un desarrollo urbano eficiente y que se pueda sostener económicamente para horizontes de tiempo de más de 50 años.

Dado que aún no se cuenta con información técnica de las obras de ampliación del aeropuerto de la Ciudad de México, esta opción de aprovechamiento no se incluyó como una opción de uso de suelo para su valoración. Sin 
embargo, consideramos una sección en la cual discutimos sus posibles repercusiones.

\section{Método}

Se utilizó un método de planeación participativa para sistematizar la opinión de los expertos invitados. Se integraron tres actividades: a) diagnóstico, en donde se identificaron los problemas, b) construcción de los escenarios deseados a futuro con el fin de c) obtener un plan para resolver la problemática.

De esta manera, en febrero de 2014 se llevaron a cabo dos talleres en los que participaron un total de 25 expertos de diferentes áreas del conocimiento: calidad del aire, calidad de suelo, hidrogeología, mecánica de suelos, manejo de residuos, energías renovables y legislación ambiental. En el primer taller se analizó la problemática ambiental del ex lago de Texcoco en particular y de la ZMVM en general. En este taller los diferentes expertos sintetizaron la información existente relacionada con los potenciales y las funciones de regulación del ex lago de Texcoco desde su área de competencia. Con base en la información compilada se analizaron, en grupos de trabajo, las opciones de uso de suelo que podrían contribuir a mitigar particularmente las emisiones de partículas suspendidas (PM) provenientes del ex lago de Texcoco. Con base en los resultados obtenidos se procedió, en un segundo taller, a describir cada opción en un grupo de trabajo específico, donde además se analizaron los beneficios, las limitaciones y los retos que implica la realización de la misma.

A continuación se sintetizan los resultados obtenidos en cada taller.

\section{Problemática ambiental de la Zona Metropolitana del Valle de México y su relación con la Zona Federal del Lago de Texcoco}

3.1. Texcoco y la gestión hidráulica en el Valle de México: Breve perspectiva histórica y situación actual

El lago de Texcoco fue el cuerpo de agua más importante del valle de México a la llegada de los españoles; todas las aguas pluviales, las de ríos y manantiales concurrían hacia él (Cruickshank, 2007). Los aportes más grandes provenían del desbordamiento de las aguas del lago Zumpango y del lago Xaltocan (laguna de San Cristóbal), provocando el aumento del nivel lacustre en el lago de Texcoco. Esto ocasionaba los problemas de las históricas inundaciones que datan desde 1450 afectando a la Gran Tenochtitlán (Cruickshank, 2007). En la época prehispánica se llevaron a cabo distintas obras para evitar el desbordamiento del lago de Texcoco como el dique de Nezahualcóyotl y el dique de Ahuízotl o San Lázaro, además de calzadas y albarradones con los que la zona lacustre quedó dividida en sectores que operaban como vasos comunicantes para regular las avenidas y controlar la calidad de las aguas.

Sin embargo, las inundaciones continuaron, lo que motivó que en la época colonial se hiciera un desagüe general de la cuenca (Ezcurra, 1990; Cruickshank, 2007). Las obras iniciaron con la construcción del Tajo de Nochistongo en 1607 (SACMEX, 2012). En 1857 se realiza el proyecto más completo que consistió en la construcción de un canal que partía de la orilla occidental del lago de Texcoco hasta el túnel Tequixquiac Núm. 1 que descargaba en el río Tula. Posteriormente, las obras de drenaje artificial continuaron, lo que llevó a la construcción del túnel Tequixquiac Núm. 2. Estos proyectos desecaron paulatinamente el lago de Texcoco hasta que su lecho quedó expuesto y sujeto a la contaminación de aguas residuales de los ríos Churubusco, La Compañía y Los Remedios (Ezcurra, 1990; Cruickshank, 2007).

En la actualidad y con el hundimiento de la Ciudad de México, el ex lago de Texcoco ha quedado hasta $3 \mathrm{~m}$ por arriba de ésta. Sin embargo, desde el Proyecto "Plan lago de Texcoco", la ZFLT continua dando servicio como vaso regulador de corrientes naturales y artificiales (SRH, 1971). En el aspecto de la regulación hidráulica, este proyecto incluyó: a) la construcción de lagos artificiales para almacenar y regular las crecientes de los ríos de oriente de la cuenca y las aguas residuales crudas y tratadas, para destinarlas a riego y usos industriales; b) obras de encauzamiento y rectificación de los ríos de la cuenca tributaria para evitar desbordamientos y reducir el riesgo de inundaciones en las zonas aledañas y c) la construcción de plantas de tratamiento de aguas residuales para el manejo y suministro de los cuerpos de agua y las áreas verdes (Cruickshank, 2007).

El drenaje artificial de la cuenca de México ha conducido al siguiente resultado paradójico: todo el esfuerzo invertido en drenar la cuenca es equivalente al invertido para obtener agua para la ZMVM (Alcocer-Durand y Escobar-Briones, 1992). Ante el aumento de la demanda de agua por el crecimiento de las zonas urbanas, cada vez se requieren mayores esfuerzos para mantener este equilibrio que además, se intensifican por los cambios de uso del suelo en las zonas de recarga de mantos acuíferos (AlcocerDurand y Escobar-Briones, 1992; Cruickshank, 2007). Las consecuencias de esta alteración son vistas no sólo en las inundaciones y en los azolves de presas y ríos sino también en la escasez de agua en la ZMVM (Cruickshank, 2007).

El agua potable para la cuenca $\left(62 \mathrm{~m}^{3} / \mathrm{s}\right)$ es abastecida principalmente de la extracción de agua subterránea que representa el $73 \%$ del total, dando lugar a la sobreexplotación de los mantos acuíferos (CONAGUA, 2011). El $27 \%$ restante proviene de trasvases de otras cuencas; así el sistema Cutzamala, aporta el $18 \%$ y se complementa con el sistema Lerma (6\%), con ríos y manantiales (3\%). El traslado del agua del sistema Cutzamala requiere además de un costoso bombeo para elevar el agua $1000 \mathrm{~m}$ para que después pueda fluir por gravedad hacia el valle de México. 
La sobreexplotación del acuífero no sólo pone en riesgo la fuente de abastecimiento de agua subterránea como fuente principal, sino que conlleva a uno de los problemas más serios que enfrenta la ZMVM: el hundimiento del suelo en todo el valle de México de manera gradual e ininterrumpida (Maderey y Carrillo, 2005; CONAGUA, 2012). En algunos puntos de la ciudad los hundimientos superan los $40 \mathrm{~cm} /$ año (CONAGUA, 2012). Este hundimiento no sólo afecta directamente la infraestructura urbana, sino también al sistema de drenaje. Esto se muestra en la Figura 1 donde se aprecia como El Gran Canal del Desagüe ahora presenta una contrapendiente con respecto al diseño original.

Además de los daños a la infraestructura debido a los hundimientos diferenciales, muchos de los flujos normales de recorrido de aguas tanto superficiales como subterráneas han cambiado, es decir, zonas que aportaban agua ahora la reciben y por lo tanto se inundan, mientras que las zonas que anteriormente recibían agua ahora se han secado o están en proceso de desecación (Escolero, comunicación personal).

De 1983 a 1992 la zona del Aeropuerto Internacional de la Ciudad de México (AICM) y, parcialmente, la ZFLT presentaban una velocidad de hundimiento de entre $20 \mathrm{y}$ $30 \mathrm{~cm} /$ año. Entre 1992 y el 2000 el hundimiento aumentó considerablemente, asociado al crecimiento de la población y de la demanda de abastecimiento de agua potable, por lo que en la zona del aeropuerto la velocidad registrada fue del orden de 25 a $35 \mathrm{~cm} /$ año, velocidad que se mantuvo en el periodo de 2000 a 2005 en esa zona y parcialmente en la ZFLT (Rodríguez-Barrón, 2010).

Para contrarrestar el hundimiento de algunas zonas de la ZFLT se ha propuesto establecer pozos de recarga. Este tipo de sistema inyecta agua debidamente tratada al subsuelo según la NOM-014-CONAGUA-2003 y la NOM015-CONAGUA-2007 a través de pozos perforados ex profeso (Mendoza-Archundia, 2012). Otras megaciudades, como la de Tokio, han logrado detener su hundimiento evitando la extracción de aguas subterráneas, mostrando que el problema de la subsidencia tiene solución mediante la recuperación de los acuíferos, la planeación territorial y el uso de materiales de construcción más ligeros (Erkens et al., 2014a, 2014b).

El abastecimiento de agua potable para la ZMVM requiere de un sistema complejo de captación, conducción y distribución del líquido, el cual opera actualmente al límite de sus capacidades físicas y económicas sin satisfacer a cabalidad la demanda de agua (Ovando-Shelley et al., 2007; Carrera-Hernández y Gaskin, 2009; Oswald, 2011). De esta manera, la ZMVM enfrenta, entre otros, el reto de satisfacer la demanda de agua de la población, reducir la sobreexplotación de los acuíferos, controlar los hundimientos del suelo y reducir los riesgos y daños de las inundaciones (CONAGUA, 2012).

3.2. Generación, uso y consumo de energía en la ZMVM

En cuanto a consumo de electricidad, el estudio del Economist Intelligence Unit (Siemens, 2010) sitúa favorablemente a la ZMVM con respecto a otros grandes centros urbanos latinoamericanos, ver Tabla 1. Sin embargo, las principales fuentes de electricidad en la ZMVM provienen de fuentes no renovables o bien, hidroeléctricas situadas a grandes distancias o con requerimientos de mantenimiento elevados. Por ejemplo, la de Infiernillo, Guerrero, con capacidad instalada de 1120 MWe (Megavatio eléctrico), se localiza en la zona de mayor riesgo sísmico del país y por tal motivo se evalúa continuamente el comportamiento dinámico de sus estructuras (ICA, 2011). La de Chicoasen, Chiapas, con capacidad instalada de $3330 \mathrm{MWe}$, se encuentra a $850 \mathrm{~km}$ de distancia de la ZMVM incurriéndose en gastos y pérdidas por transmisión. Según algunos documentos, en México las pérdidas por transmisión ascienden hasta en un 17.8 \% (CMIC, 2013).

En la ZMVM no existe aún aprovechamiento de las fuentes de energía renovable para generar electricidad de consumo público a nivel local, a pesar de que su precio por $\mathrm{kW}$ es cada vez más competitivo, ver Tabla 2. Cabe destacar que al generarse electricidad localmente por fuentes renovables se evitarían costos de instalación y mantenimiento de líneas de transmisión a larga distancia aunque por su intermitencia, como la solar, se debería tener una red local de distribución e interconexión adaptable. La ZMVM por su altitud y latitud se encuentra en una situación privilegiada para captar radiación solar. La irradiancia solar en el Aeropuerto Internacional de la Ciudad de México es similar a la de Phoenix, Arizona, con un promedio anual de 19 MJ por $\mathrm{m}^{2}$ diario (Mondragón-Rodríguez, 2013; Solar Irradiance, 2014). Cabe señalar que el estado de Arizona es una de las zonas de mayor interés para el desarrollo de energía solar de los EEUU (FUMEC, 2014). Además se generan desechos sólidos que se podrían utilizar para generar electricidad, a partir de biogás, como lo propone Aguilar-Virgen et al. (2014) para Baja California, o como se hace actualmente en Monterrey, NL, a través de Bioenergía de Nuevo León S.A. de C.V., con una capacidad de $12 \mathrm{MWe}$ (GENL, 2014).

La ZMVM puede promover el uso de fuentes renovables

Tabla 1. Energía y $\mathrm{CO}_{2}$. Se considera el total anual de emisiones de $\mathrm{CO}_{2}$ en kg y el total anual de consumo de electricidad en MJ por unidad de PIB (en miles de dólares). Tomado de: Siemens, 2010.

\begin{tabular}{lllll}
\hline $\begin{array}{c}\text { Muy por } \\
\text { debajo del } \\
\text { promedio }\end{array}$ & $\begin{array}{c}\text { Debajo del } \\
\text { promedio }\end{array}$ & Promedio & $\begin{array}{c}\text { Encima del } \\
\text { promedio }\end{array}$ & $\begin{array}{c}\text { Muy por } \\
\text { encima del } \\
\text { promedio }\end{array}$ \\
\hline \multirow{6}{*}{ Guadalajara } & Belo & Borizonte & Bogotá & \\
& Medellín & Brasilia & Curitiba & \\
& Montevideo & Buenos Aires & Ciudad de & São Paulo \\
Santiago & Porto Alegre Lima & Méco & \\
& Puebla & Monterrey & Rio de & \\
& & Quito & Janeiro & \\
\hline
\end{tabular}


Conducción por gravedad al Gran Canal de Desagüe

Sistema de bombeo al Gran Canal de Desagüe

Sistema de bombeo al Gran Canal de Desagũe

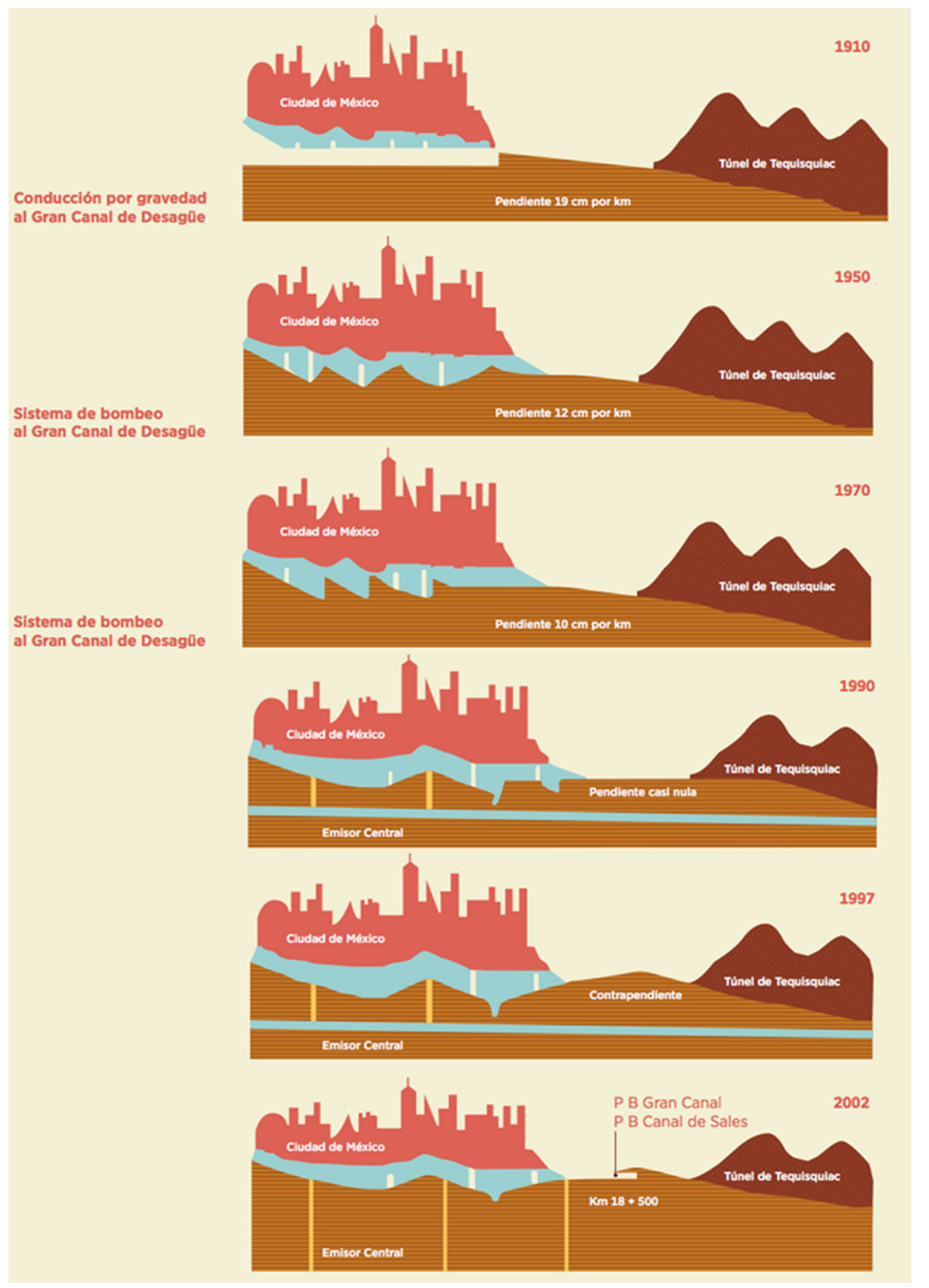

Figura 1. Hundimiento de la Ciudad de México originando la presencia de una contrapendiente en el sistema de colectores del drenaje profundo. Tomado

de: SACMEX (2012).
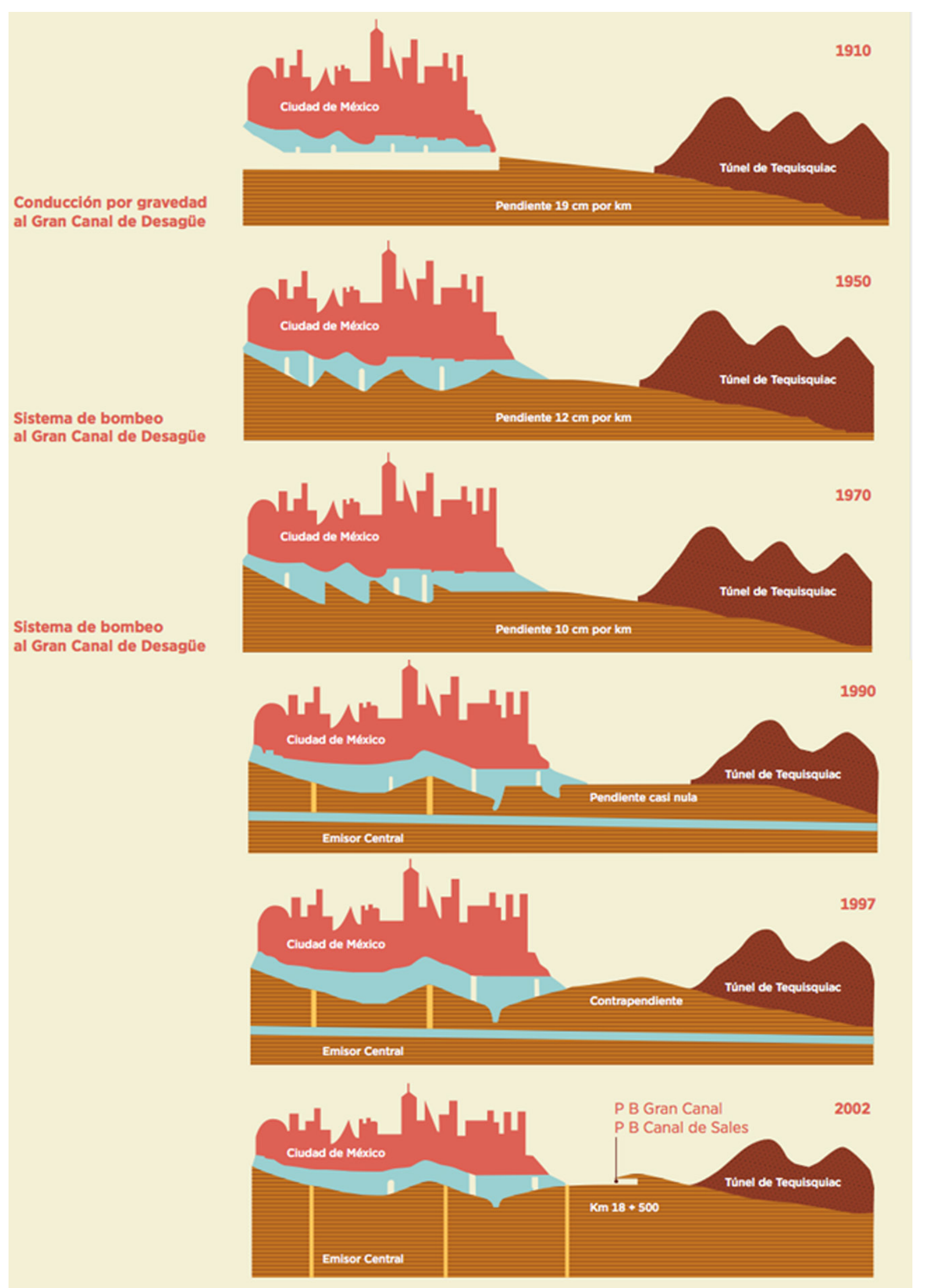
Tabla 2. Costos nivelados de producción de electricidad por fuente en los EEUU. Adaptado de EIA (2014).

\begin{tabular}{lc}
\hline \multicolumn{1}{c}{ Tipo de Planta Eléctrica } & $\begin{array}{c}\text { Costo Nivelado de } \\
\text { Producción Eléctrica } \\
\text { (Dlls./MWh) }\end{array}$ \\
\hline Carbonífera convencional & 95.6 \\
$\begin{array}{l}\text { Gas natural ciclo combinado } \\
\text { convencional }\end{array}$ & 66.3 \\
$\begin{array}{l}\text { Gas natural con turbina de } \\
\text { combustión convencional } \\
\begin{array}{l}\text { Renovable: aprovechamiento de } \\
\text { biomasa } \\
\text { Renovable: Solar Fotovoltaico }\end{array} \\
\text { Renovable: Solar Termal }\end{array}$ \\
\hline
\end{tabular}

de energía. Por ejemplo, 29 estados de los EEUU fijan metas para que al menos $10 \%$ de su energía eléctrica provenga de fuentes renovables (EIA, 2012). Generar energía renovable en la ZMVM estaría de acuerdo con estas tendencias y las fijadas por la Ley de Cambio Climático que proyecta que la oferta eléctrica de estas fuentes sea de hasta un $35 \%$ para el 2026 (SENER, 2012). De comprometerse con esta tendencia localmente, permitiría a la zona económicamente más importante del país enfrentar un futuro energético mundial incierto con fuentes de energía diversificadas, aprovechando su propio crecimiento y su situación geográfica con tecnologías ya existentes.

En cuanto a electricidad generada mediante termoeléctricas, la proveniente del Complejo de Tula usa combustóleo. Sus emisiones inciden negativamente en la calidad del aire de la ZMVM principalmente en las concentraciones de $\mathrm{SO}_{2}$ durante los meses de septiembre a octubre (García-Escalante et al., 2014).

En la ZMVM se consumen 9600 millones de litros al año de gasolina y diésel, es decir, casi 27 millones de litros al día, principalmente para el transporte (SEDEMA, 2012). Al no contar con fuentes "limpias" suficientes de generación eléctrica no se pueden aprovechar las ventajas ambientales de las nuevas tecnologías de transporte público y privado eléctrico: si la electricidad generada para cargar sus baterías o mover sus motores proviene de fuentes con alto contenido de carbono, las emisiones netas de gases de efecto invernadero superan a las de transportes que usan combustible fósil convencional (Babaee et al., 2014). Toda planificación a futuro de cualquier ciudad moderna debe contemplar esta posibilidad. Hay que subrayar que el transporte eléctrico tiene una mayor eficiencia energética (Delucchi et al., 2014): según el Departamento de Energía de los EEUU, un vehículo eléctrico convierte 59 - $62 \%$ de la energía eléctrica de la red a motriz, mientras que uno convencional convierte $17-21 \%$ de la energía del combustible en motriz (Fuel Economy, 2014), además de requerir de menos mantenimiento. Por estas razones se incrementa su importancia a nivel mundial señalando una tendencia, aunque en el caso privado aún ocupa un porcentaje pequeño de ventas por sus precios todavía altos. Estudios realizados en México, señalan que el uso de automóviles con tren motriz eléctrico tiene importantes beneficios energéticos y en salud pública (Jazcilevich et al., 2011).

A medida que tecnologías más eficientes de iluminación pública y residencial sean introducidas en el mercado, como ha sucedido con la sustitución de focos incandescentes en EEUU y en la Comunidad Europea por LEDs, la generación de energía eléctrica mediante fuentes renovables en la ZMVM puede tener una mayor cobertura. La regeneración de la ZFLT que incluya generación de electricidad y calor mediante fuentes renovables intermitentes, como la solar, o no intermitentes, como la producida a partir de residuos sólidos orgánicos urbanos, aunada a políticas de ahorro energético, puede contribuir a la diversificación de fuentes de energía aprovechando recientes logros tecnológicos en la materia.

Todas las propuestas presentadas aquí, generan electricidad de fuentes renovables para su autoabastecimiento, además de generar electricidad regional a partir de energía solar y/o a partir de residuos sólidos orgánicos urbanos.

\subsection{Manejo de residuos sólidos orgánicos urbanos}

La generación de residuos sólidos urbanos se ha incrementado notablemente en los últimos años. Tan sólo entre 2003 y 2011 creció $25 \%$, como resultado principalmente del crecimiento urbano, el desarrollo industrial y el cambio en los patrones de consumo (SEMARNAT, 2013). En cuanto a su composición, los residuos sólidos urbanos también han cambiado de manera importante en las últimas décadas en el país, proceso que no está acompañado de políticas internas y de largo plazo orientadas a procesar o a reciclar lo generado (IglesiasPiña, 2007; SEMARNAT, 2013). En la década de los 50, el porcentaje de residuos orgánicos en la basura oscilaba entre 65 y $70 \%$ de su volumen, mientras que para 2011 esta cifra se redujo al 52.4\% (SEMARNAT, 2013).

Por otra parte, la generación total de residuos sólidos urbanos difiere de manera importante a nivel nacional. Por ejemplo en 2011 se produjeron alrededor de 41 millones de toneladas, equivalente a 112.5 mil toneladas diarias. De este total, la región centro contribuyó con el $51 \%$ de la generación y por entidad federativa, las que produjeron los mayores volúmenes de residuos sólidos urbanos en 2011 fueron el Estado de México (16\% del total nacional) y el Distrito Federal (12\%) (SEMARNAT, 2013).

El incremento de los residuos ha saturado algunos de los espacios de confinamiento y los que están en operación, carecen de infraestructura básica, generándose problemas de contaminación. Sin embargo, estos residuos tienen un 
alto valor ya que más de la mitad pueden ser aprovechados. Ejemplo de ello es la fracción orgánica de la cual se puede extraer biogás (gas combustible que, en su mayoría, constituye una mezcla de $\mathrm{CO}_{2}$ y $\mathrm{CH}_{4}$ ), generar abonos orgánicos e inclusive producir electricidad (Iglesias-Piña, 2007; Moreno y Meixueiro, 2007; GODF, 2010).

El Gobierno del Distrito Federal opera la planta de composta Bordo Poniente en la ZFLT que, con más de 30 ha, constituye la más grande de la República Mexicana. En el 2012 recibió aproximadamente 845434 t de residuos orgánicos al mes y produjo $162323 \mathrm{t}$ de composta, reduciendo casi en un $80 \%$ la cantidad de los residuos destinados a confinamiento. La composta que se produce se utiliza en los parques y jardines de las delegaciones del Distrito Federal, en las áreas verdes de escuelas públicas, y para sanear las celdas del relleno sanitario Bordo Poniente (GODF, 2010; Tetra Tech ES Inc, 2013).

A pesar de la producción de composta y su posterior empleo en zonas verdes, falta desarrollar un mercado para colocar la totalidad de ésta. También urge establecer una normatividad que regule las cantidades máximas de aplicación por superficie y lapso de tiempo, para evitar posibles efectos negativos en la calidad del agua subterránea y del aire. Además, la cantidad de residuos sigue incrementándose, por lo que la reducción de los mismos debe contemplar otras medidas, como la explotación y aprovechamiento de biogás, planteadas en el "Convenio de Coordinación para el Cierre y Clausura Definitiva de la IV Etapa del Relleno Sanitario Bordo Poniente" y que hasta la fecha no han sido implementadas (CONAGUA, 2010; GODF, 2010; Estrada-Núñez, comunicación personal). No obstante, este tipo de procesamiento no es el único que puede reducir los desechos orgánicos y al mismo tiempo dar un beneficio adicional como la producción de biocombustibles y energía eléctrica. Como ejemplo se tiene que en Baja California cuatro comunidades tienen el potencial de producir $68.69 \mathrm{~m}^{3} / \mathrm{t}$ de metano a partir del biogás generado en los sitios de disposición final, lo cual representa $760492.8 \mathrm{MWe} \mathrm{h}^{-1}$ en 17 años (Aguilar-Virgen et al., 2014). Adicionalmente, la ciudad de Monterrey opera una planta generadora de electricidad de $12 \mathrm{MWe}$ a partir de biogás metano obtenido de residuos sólidos orgánicos urbanos (GENL, 2014).

A nivel internacional, la Figura 2 muestra el destino que se les da a los residuos sólidos urbanos en diferentes países. Cabe destacar que en Japón, tan sólo el 2 \% finaliza en un relleno sanitario. Las propuestas que aquí se presentan para la ZFLT abren la posibilidad no sólo de seguir utilizando tecnologías para el tratamiento de residuos sino, aún más, de ampliarlas.

Si bien la incineración de residuos sólidos urbanos no está permitida legalmente en México, el reciclado y disposición final, con generación de energía a partir del biogás, son alternativas que tienen potencial en la ZMVM y que pueden hacer frente a los retos que representa el hecho de manejar adecuadamente la cantidad de residuos que se generan, por lo que cabría reconsiderar su legalización bajo un marco normativo adecuado.

\subsection{Calidad del aire de la ZMVM}

El ex vaso de Texcoco ha sido una fuente de PM (Material Particulado, siglas en inglés) desde que las obras de drenaje de la cuenca de México llevaron a desecar el antiguo lago de Texcoco. La desaparición del espejo de agua dejó una superficie cubierta de sedimentos con altas concentraciones de sales solubles que impiden el desarrollo de una cobertura vegetal (Cruickshank, 2007).
E.U.(2008)

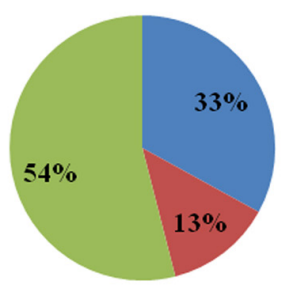

Japón (2007)

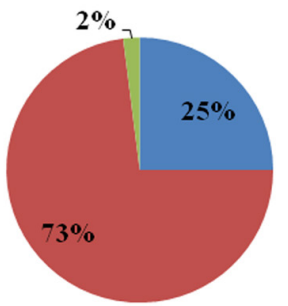

- Reciclado

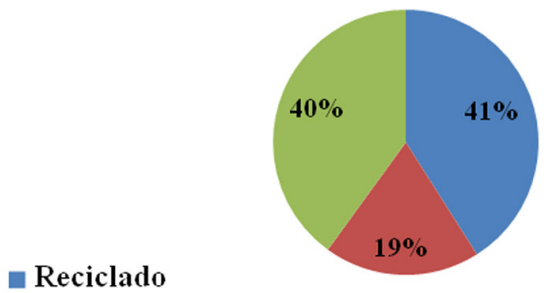

- Incineración

Relleno sanitario
Corea (2007)

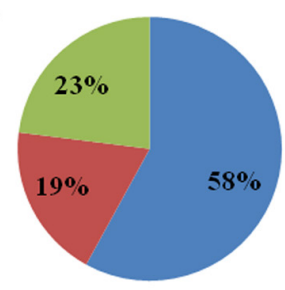

Figura 2. Porcentaje internacional de uso de residuos sólidos urbanos. Basado en Sakai et al. (2011). 
Particularmente en los años 1970s se reconoció que esta zona era fuente de una cantidad importante de partículas a la atmósfera, por lo cual se inició un ambicioso plan para vegetar estos terrenos y así estabilizarlos (Plan Lago de Texcoco). A más de 40 años de su inicio se registran avances importantes, pero aún hay grandes superficies desprovistas de vegetación (Fernández-Buces, 2006).

Actualmente la emisión de material por erosión eólica es uno de los responsables de los episodios de contingencia por PM con diámetro aerodinámico menor a 10 micrómetros $\left(\mathrm{PM}_{10}\right)$ en el valle de México, afectando a millones de habitantes. Recientemente se reportan aumentos extraordinarios de $\mathrm{PM}_{10}$ llegando a concentraciones de más de $300 \mu \mathrm{g} / \mathrm{m}^{3}$, lo que supera el índice IMECA de más de 60 puntos de PM y la calidad del aire se clasifica como extremadamente mala. Esto afecta a regiones densamente pobladas del noreste y sureste de la ZMVM. Ejemplos de ello son mayo 05, 2012 y junio 11, 2012 en Tláhuac y Villa de las Flores, respectivamente (SIMAT, 2014a, 2014b). Al estar sujeta la ZMVM al transporte de material particulado por erosión se explica por qué en la Ciudad de México el 61 $\%$ de material $\mathrm{PM}_{10}$ y el $16 \%$ de $\mathrm{PM}_{2.5}$ sea de origen mineral, como se aprecia en la Figura 3. Estos eventos erosivos ocurren durante la temporada de secas, especialmente en los meses de diciembre a marzo.

Como muestran los trabajos de Díaz-Nigenda et al. (2010), el ex vaso de Texcoco sigue siendo una fuente importante de partículas suspendidas a la atmósfera. En la Figura 4 se presentan resultados de un modelo acoplado de meteorología y erosión (Díaz-Nigenda et al., 2010). Se muestra un episodio de emisión por erosión en la zona del ex vaso, y lo que ocurre cuando estas emisiones son mitigadas al humedecer el suelo. Esto indica que el mitigar las emisiones provenientes de la ZFLT reduciría sustancialmente los días con concentraciones extraordinarias de PM, aunque no son las únicas fuentes.

\section{Opciones de uso de suelo: beneficios, limitaciones y retos en la ZFLT}

Se generaron propuestas conceptuales incluyendo su factibilidad desde el punto de vista técnico y legal. Además se discute el balance energético de cada una así como sus beneficios ambientales. Todas ellas deben someterse a etapas de pruebas experimentales para constatar su efectividad antes de realizarse. Las etapas experimentales se describen al final de cada propuesta; consisten de proyectos piloto a baja escala. Las mediciones obtenidas determinarán la viabilidad técnica de la propuesta y su interacción con las demás.

Las siguientes son las opciones de uso que se analizaron:

- Rehabilitación de suelos y vegetación.

- Regeneración de zonas lacustres.

- Manejo de residuos sólidos orgánicos.

- Generación de electricidad y calor con energía solar.

Las propuestas se han denominado de esta manera para poder explicar por separado sus rasgos más importantes desde el punto de vista tecnológico y de viabilidad legal, pero también se enfatiza su capacidad de interactuar e integrarse entre ellas, como se explicará cuando se les describa en su correspondiente sección.

\subsection{Rehabilitación de suelos y vegetación en la ZFLT}

El origen de la extrema salinidad en la ZFLT se debe a procesos de intemperismo químico de las rocas volcánicas y a los flujos de agua que han concentrado a estos iones en la parte más baja de la cuenca. Por otra parte, la desecación del lago de Texcoco por las obras de drenaje para evitar las inundaciones de la Ciudad de México, han contribuido a la acumulación de estas sales en el acuitardo superficial conformado por los materiales arcillosos de los depósitos lacustres (SRH, 1971; Cruickshank, 2007).

Para la rehabilitación de los suelos y el posterior establecimiento de una cubierta vegetal, a partir de los años
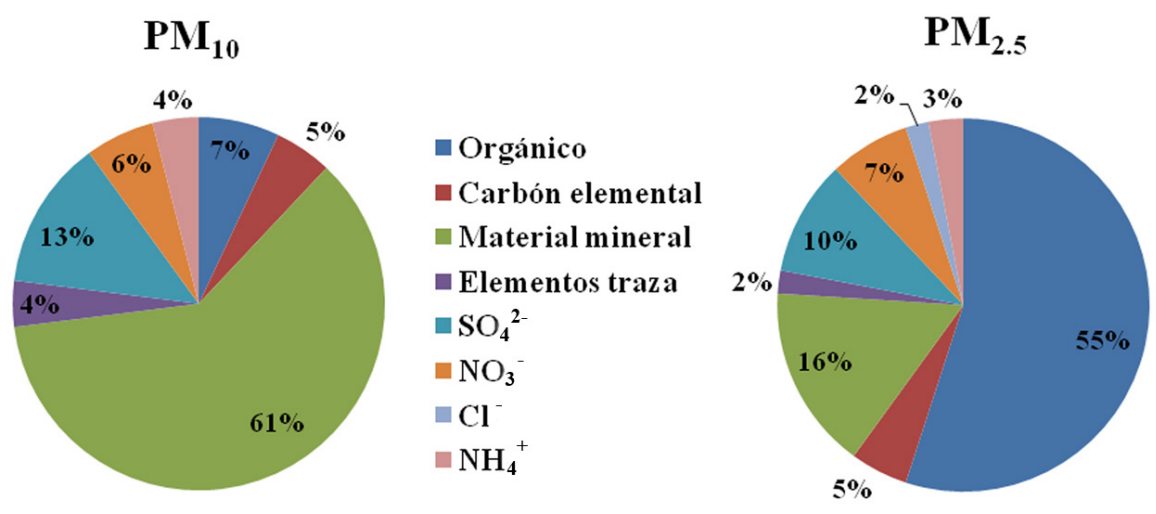

Figura 3. Caracterización química de $\mathrm{PM}_{10} \mathrm{y} \mathrm{PM}_{2.5}$ en el Instituto Mexicano del Petróleo, Ciudad de México, durante la campaña MILAGRO, 2006. Basado en Querol et al. (2008). 
a)

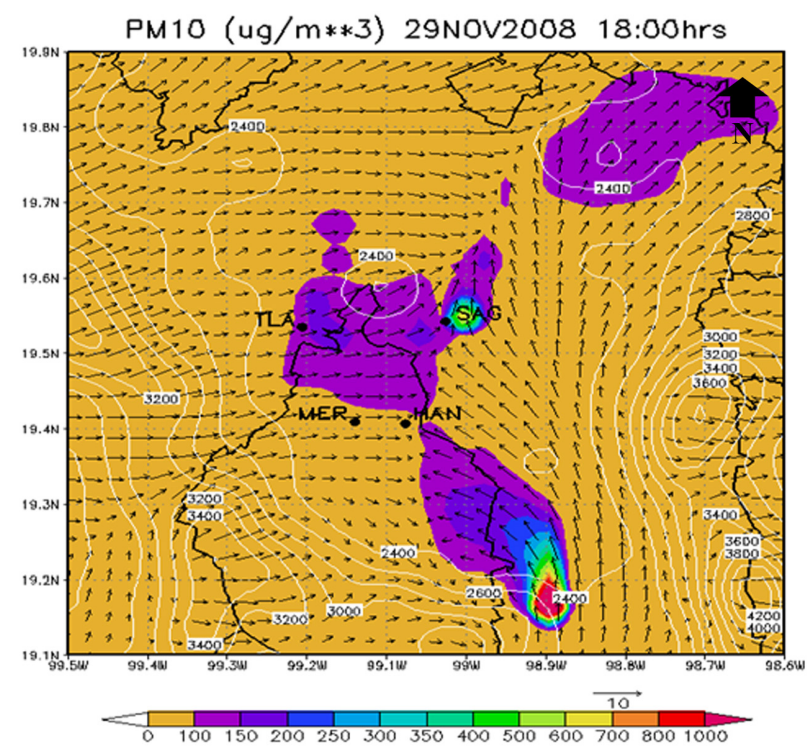

c)

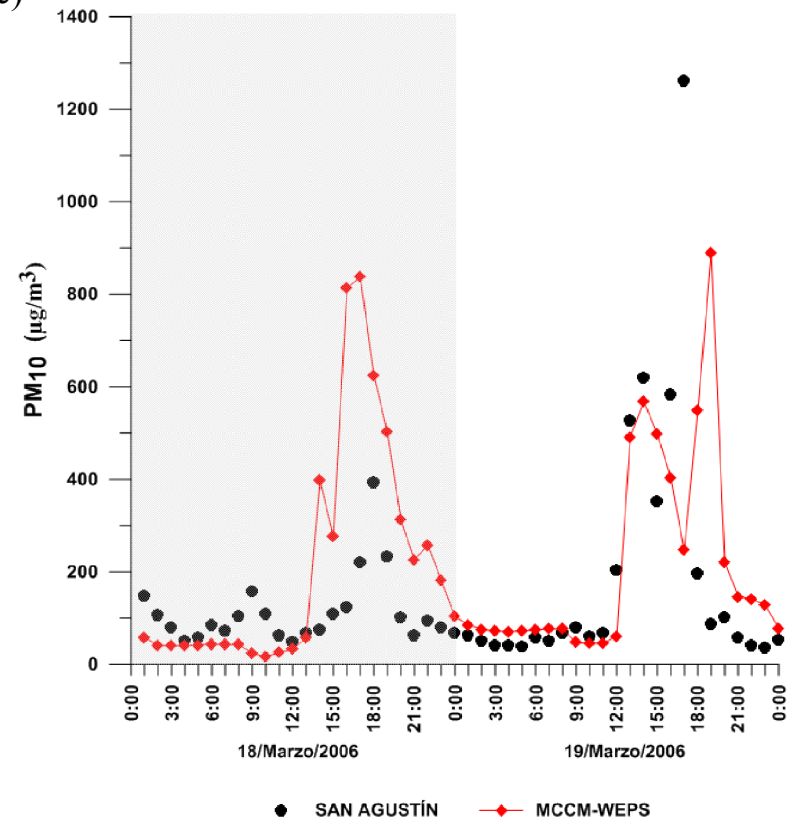

b)

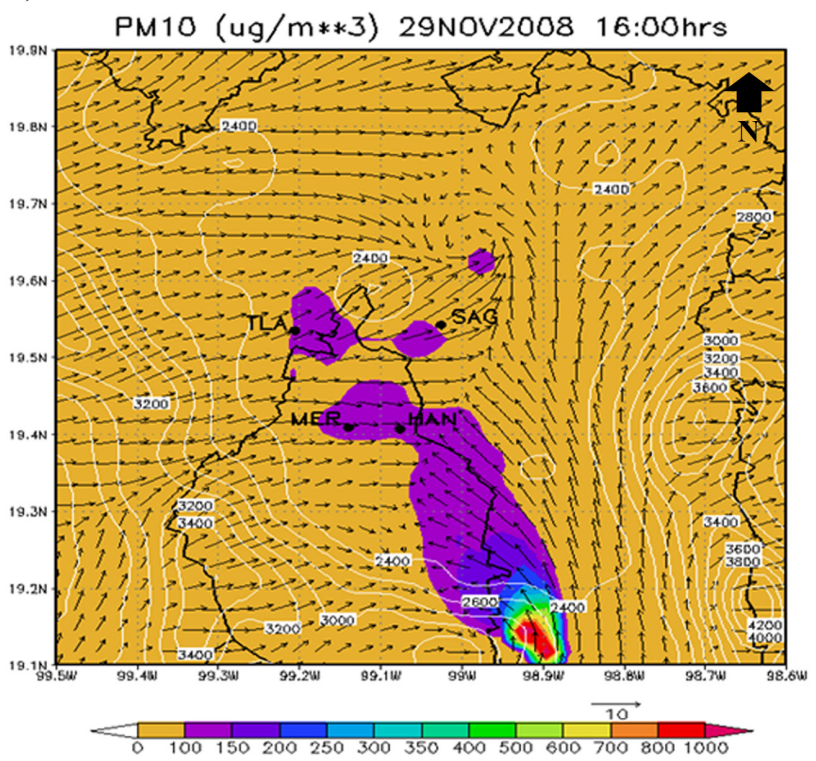

d)

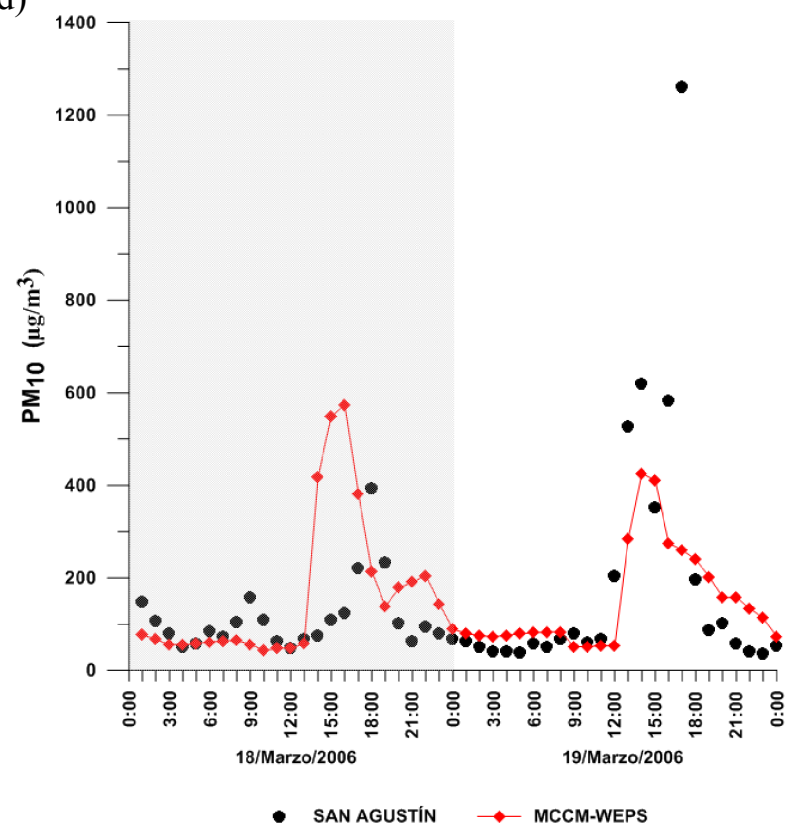

Figura 4. En (a) y (b) la distribución geográfica de $\mathrm{PM}_{10}$ en el Valle de México para un escenario modelado actual y con lago, respectivamente. En (c) y (d) estas concentraciones en la Estación San Agustín (SAG). Con puntos las mediciones por la RAMA, y con líneas los resultados de la modelación. Elaboración de: Díaz-Nigenda.

1970s se realizaron obras para abatir el nivel freático, y se aplicaron láminas de lavado y drenajes parcelarios para reducir el exceso de sales (CONAGUA y UAch, 2004; Cruickshank, 2007). Se consideró el uso de aguas negras, ya que su contenido de materia orgánica podría ayudar a mejorar los suelos, sin embargo, gran parte de las aguas negras del Distrito Federal estaban concesionadas para uso agrícola (SRH, 1971). También se llevaron a cabo pruebas con aplicación de corrientes eléctricas y adición de mejoradores químicos como yeso, azufre y caliza (SRH, 1971).
En algunas áreas se obtuvieron resultados positivos en cuanto a la disminución de las altas concentraciones de sales y sodio. Fernández-Buces et al. (2009) documentaron que las obras realizadas en 30 años disminuyeron la salinidad en la parte sur y oriente de la antigua planicie lacustre, pero las áreas topográficamente más bajas, localizadas en la zona oeste y noroeste, presentan ahora mayores contenidos de sales solubles (Figura 5). En estas zonas el nivel freático se encuentra muy cercano de la superficie, lo cual impide su habilitación usando drenaje parcelario y lavado de sales por irrigación, dificultando y elevando los costos de la 
rehabilitación.

La alta salinidad tiene diferentes efectos en la supervivencia y crecimiento de la vegetación; una de ellas es la deshidratación de las plantas, es decir, la alta concentración de solutos en el suelo eleva el potencial osmótico del suelo y con ello extrae el agua de las raíces evitando así, la absorción de este líquido por la planta. Por otra parte, las sales también pueden tener un efecto tóxico por los iones presentes en el suelo (por ej. el $\mathrm{Na}$ ) y además ocasionar deficiencias de otros nutrimentos como $\mathrm{K}, \mathrm{Ca}, \mathrm{Zn}$,
Fe o $\mathrm{Cu}$, lo que se traduce en una disminución de las tasas de crecimiento dependido de la fase del ciclo de vida de la planta. En cuanto a las propiedades del suelo, la sodicidad puede llevar a la pérdida de estructura y por consiguiente ocasionar deficiencias en la aireación del suelo y en la oxigenación de las raíces (Tan, 1993; Brady y Weil, 2002). Por lo tanto, el abatimiento de los efectos negativos de la salinidad y la sodicidad sobre la vegetación es crucial si se pretende establecer una cubierta vegetal en suelos con altas concentraciones de sales ricas en sodio.

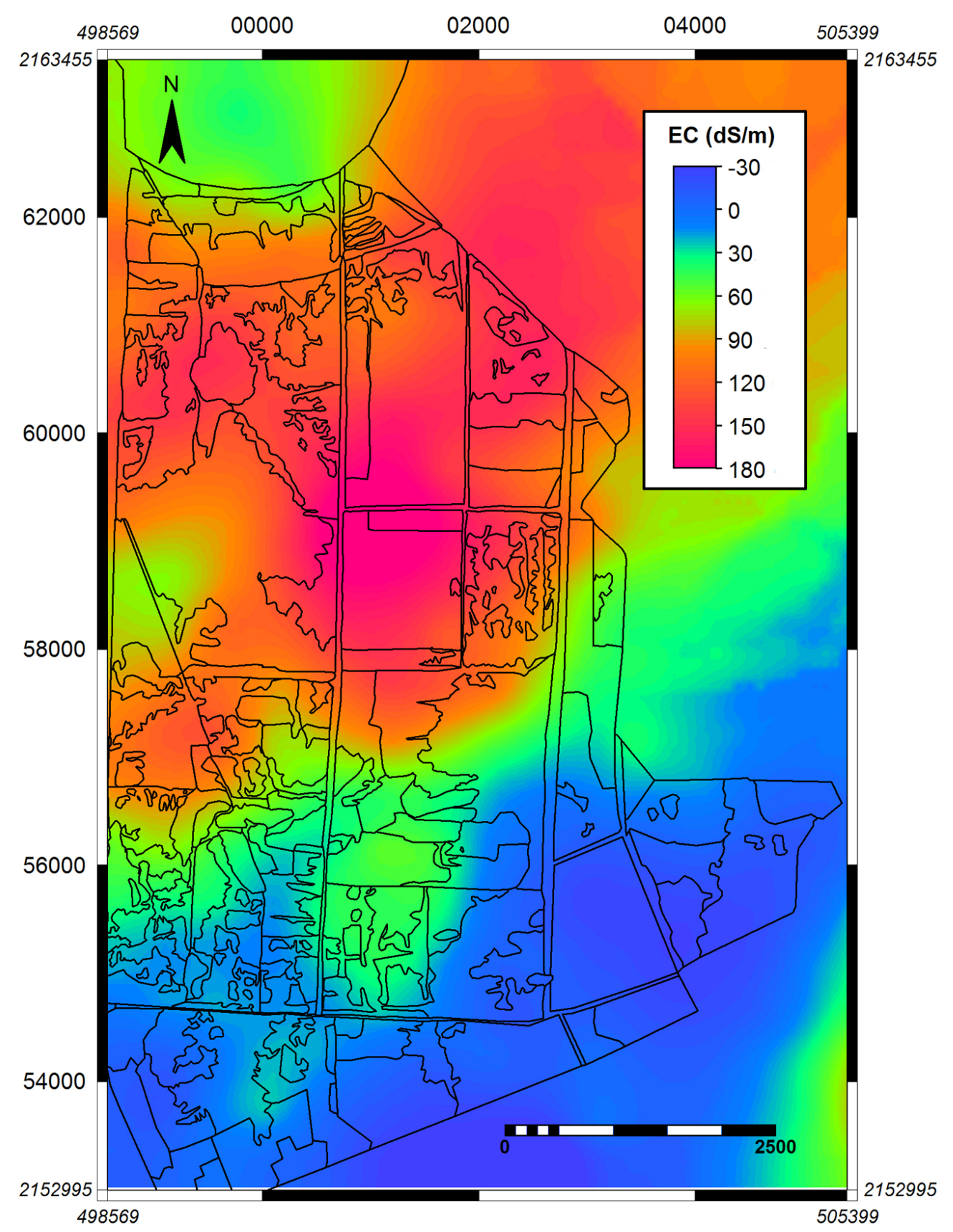

Figura 5. Mapa de cambios en la conductividad eléctrica del suelo estimada a partir de muestreos llevados a cabo en 1971 y 1998 , después de 27 años de manejo en el ex lago de Texcoco. Tomado de: Fernández-Buces et al. (2009). 


\subsubsection{Una cobertura con vegetación en la ZFLT}

La facilitación del establecimiento y desarrollo de algún tipo de vegetación en zonas carentes de cobertura implica evitar el ascenso capilar del agua salina, por un lado y, mejorar la capacidad de retención de agua aprovechable por las plantas, por el otro. Para lo primero se propone crear una capa o estrato de granulometría contrastante respecto a los sedimentos lacustres, para evitar el ascenso capilar de la salmuera, por ejemplo usando residuos de la construcción, como el cascajo. Sobre esta capa se sugiere implementar un sustrato con mayor capacidad de retención de humedad y mayor macro y mesoporosidad. Para esto se podrían usar residuos orgánicos composteados, aunque también se podría evaluar aplicar materiales orgánicos más estables tipo biocarbón. El biocarbón es un material sólido obtenido a partir de la conversión termoquímica de la biomasa en un ambiente limitado en oxígeno $(<2 \%$ ) (Lehmann, 2007; Verheijen et al., 2010; IBI, 2012). En este proceso las sustancias orgánicas son descompuestas a temperaturas que van desde los 350 hasta los $1000{ }^{\circ} \mathrm{C}$ convirtiendo la biomasa en una fracción de carbono estable (EBC, 2013).

Los residuos orgánicos composteados se mineralizan más rápidamente que el biocarbón, por lo que pueden contribuir en la emisión de $\mathrm{CO}_{2}$ y de $\mathrm{NH}_{3}$ a la atmósfera; mientras que el biocarbón, al ser más estable tiene efectos más duraderos en la estructura del suelo y además puede retener las sales por adsorción en sus superficies. Es importante cuantificar la mineralización de la materia orgánica antes y después de la aplicación de la composta y del biocarbón al suelo, para asegurar tanto la disminución en la concentración de sales y sodio como los efectos en la rehabilitación del suelo para el establecimiento de la cubierta vegetal.

\subsubsection{Viabilidad técnica y legal en la ZFLT}

La factibilidad de esta propuesta requiere de analizar la disponibilidad y los costos de transporte de cascajo u otros residuos de granulometría gruesa para formar la barrera física que evite el ascenso capilar de la sal. Además, es necesario considerar la disponibilidad de aditivos orgánicos como composta y/o biocarbón a aplicar, que se podrían obtener de una planta de gasificación, como se propone en la propuesta de "Manejo de residuos sólidos orgánicos" y de "Generación de electricidad y calor con energía solar", o bien, de la planta de composta ya en operación. Además, es importante evaluar las necesidades de agua para el lavado de sales y el mantenimiento de la cobertura durante la época seca, así como la disponibilidad y la calidad del agua, su origen y la normatividad respectiva. Esto puede conjuntarse con las propuestas de "Regeneración lacustre", "Manejo de residuos sólidos orgánicos” y "Generación de electricidad y calor con energía solar".

En cuanto a las poblaciones animales asociadas a la zona y al pasto salado, Distichlis spicata (insectos, aves, roedores, etc.) se requiere contemplar la dinámica poblacional de la fauna la cual pudiera ocasionar un posible efecto plaga. Finalmente, los esfuerzos de pastización y movilidad de las sales de 2000 a la fecha deben ser monitoreados por medio de mapas de análisis espacio-temporal.

Respecto de la factibilidad legal, se considera que la propuesta puede cumplir con los requerimientos legales para su ejecución si se toman en consideración las siguientes premisas:

- $\quad$ El tipo de sustrato a utilizar depende en gran medida el tratamiento jurídico, así como de las autoridades responsables encargadas de dicha regulación. Por ejemplo, en caso de que el sustrato sea un residuo de manejo especial, como lo es el cascajo u otro de características similares, la competencia será estatal y se requerirá un plan de manejo;

- La autorización de uso de zona federal, independiente del tipo de sustrato a utilizar, que deberá sujetarse a lo previsto en la Ley de Aguas Nacionales y su reglamento; $y$,

- La elaboración de una manifestación de impacto ambiental o estudio preventivo, según lo requiera la autoridad competente (Federación) que incluya los alcances del proyecto así como las medidas de mitigación para los impactos que se pudieran generar derivados del proyecto.

Queda claro que cualquier obra o actividad en la zona requerirá obtener las concesiones, autorizaciones y permisos de distintas autoridades, la mayoría concentradas en el orden federal.

Por último, respecto de la generación de energía eléctrica, se deberá tener en consideración las nuevas regulaciones en el sector derivadas de la reciente reforma energética.

\subsubsection{Balance Energético en la ZFLT}

Esta propuesta podría conjuntarse con la de "Manejo de residuos sólidos orgánicos" y de "Generación de electricidad y calor con energía solar" para obtener energía calorífica para la purificación de agua mediante evaporación y condensación, o con la propuesta de "Regeneración lacustre", que propone un sistema de purificación continuo. Esta agua se usaría para el riego de la vegetación que evitaría la erosión de los suelos en el ex lago, o bien, podría usarse para la producción de verduras en invernaderos, por medio de un sistema hidropónico. Además se podría contar con energía para las necesidades de temperatura, iluminación, aireación y sistemas riego del invernadero, así como, para implementar sistemas de bombeo de agua y de drenaje en las zonas donde se logre la pastización.

Las zonas que, por su alta salinidad y sodicidad, resulten poco pertinentes para mantener una cubierta vegetal, se podrían destinar para la recuperación de lagos artificiales en los cuales se podrían a su vez localizarlos sistemas de generación de electricidad fotovoltaica con paneles solares flotantes. 


\subsubsection{Beneficios ambientales}

Si se logra establecer una cobertura vegetal en las zonas desprovistas de la misma, no sólo se tendría una reducción de partículas emitidas a la atmósfera, sino que también se podría crear un sumidero de carbono, además de la consecuente creación de empleo, regulación del microclima, aprovechamiento de residuos orgánicos y de la construcción, y el reciclaje de nutrimentos.

\subsubsection{Desarrollo de la fase experimental en la ZFLT}

Para conocer la factibilidad de esta opción se propone el diseño e instalación en el sitio de una capa o barrera física probando diferentes materiales o residuos de la construcción como cascajo para inhibir el ascenso capilar del agua freática salina. Al respecto se tendría que calcular el espesor de la capa de cascajo, el cual dependerá de la distancia al manto freático.

También se tendría que contemplar la adición de diferentes tipos de abonos orgánicos como la composta producida en la planta de composta Bordo Poniente y biocarbón, posiblemente producido también en la planta de composta. Además de medir sus efectos colaterales como la emisión de gases con efecto invernadero y la captura de carbono en el suelo en forma de biomasa microbiana, humus, y raíces.

A la par se evaluaría la supervivencia y el crecimiento de Distichlis spicata, especie nativa y dominante de la ZFLT y altamente tolerante a la sal y a la anegación (Rzedowski y Rzedowski, 2005; Fernández-Buces et al., 2006). Finalmente la fase experimental debería contemplar la regulación de inundaciones en época de lluvias, es decir, saber cuánto tiempo toleran las plantas el anegamiento.

Para calcular la necesidad de electricidad y calor de las zonas revegetadas y del complejo de invernaderos se montarían sistemas piloto de generación de electricidad según las propuestas de "Manejo de residuos sólidos orgánicos" y de "Generación de electricidad y calor con energía solar". Con estos sistemas y con cálculos acerca de los requerimientos energéticos para el riego, bombeo y purificación de agua se obtendrían los balances de energía que se necesiten en cada proceso. Una vez obtenidos estos datos y resultados de los experimentos, se procedería al escalamiento de la propuesta.

4.2. Regeneración lacustre mediante sistema de lagos en la ZFLT

La existencia y operación del Lago Nabor Carrillo (LNC) desde 1985 es una demostración de que es posible regenerar la ZFLT mediante un sistema de lagos. El LNC tiene una capacidad de 36 millones de metros cúbicos de agua y una superficie de 917 ha con una profundidad media de $2.3 \mathrm{~m}$ (Cruickshank, 2007; DUMAC, 2005). Para su construcción se aprovechó la compresibilidad de los suelos arcillosos del ex lago: se colocó una batería de 180 pozos de bombeo dispuestos alrededor de la futura depresión con el fin de extraer agua y provocar el hundimiento del piso. Se obtuvieron así 12 millones de metros cúbicos de capacidad inicial. Al ser llenado el lago, el peso del agua produjo un hundimiento adicional de $1.5 \mathrm{~m}$ obteniéndose finalmente su capacidad actual.

El LNC recibe el agua proveniente de una planta de tratamiento ubicada en el costado suroeste del lago, en la cual se tratan $700 \mathrm{~L} / \mathrm{s}$ de aguas negras provenientes principalmente del Río Churubusco (Maya, 2000; DUMAC, 2005). Su funcionamiento consiste en el tratamiento a nivel secundario, mediante la utilización de lodos activados convencionales y aeración mecánica superficial. El efluente de la planta de tratamiento no recibe ningún tipo de desinfección, ya que de forma natural se espera que mejore de la calidad microbiológica en el lago. Según Maya (2000) no se ha logrado la calidad del agua para fines de riego, actividades recreativas de remo, canotaje, pesca deportiva y piscicultura, actividades que originalmente se habían planeado para este lugar.

\subsubsection{Terrazas de lagos en ZFLT}

Se propone un sistema de lagos constituidos en forma de terrazas que conformarían un área de aproximadamente cuatro lagos similares aparte del ya existente LNC, o sea, un total de 4,600 ha de zona lacustre. Una representación gráfica de la propuesta se muestra en la Figura 6. Se utilizaría la misma técnica de construcción que ya ha resultado exitosa para formar cada terraza. El sistema de lagos se situaría fuera de la zona de Casa Colorada, zona destinada actualmente para desfogue de agua durante la época de lluvias y se mantendría en operación la planta de composta.

Las alturas de las terrazas y su superficie estarían sujetas a la mecánica de suelos dentro de la ZFLT. El agua se bombearía a la terraza más alta y por gravedad se formaría un ciclo continuo formando un sistema circulatorio de agua. Se llevaría a cabo un tratamiento de purificación en varios segmentos del ciclo. De esta manera el agua terminaría por ser tratada varias veces, lográndose el mejoramiento de su calidad después de varios ciclos. Para mantener el flujo sólo se compensaría la merma de volumen de agua para equilibrar los niveles de evaporación u otras pérdidas, como infiltración.

En las zonas libres de agua, se utilizarían elementos de la propuesta de rehabilitación de suelos para establecer una cobertura vegetal.

\subsubsection{Viabilidad técnica y legal en la ZFLT}

Si se multiplica por cuatro el caudal de agua proveniente del Río Churubusco utilizado actualmente para mantener el LNC, se sostendría la operación del sistema propuesto.

La factibilidad legal de esta propuesta requiere que se considere lo siguiente:

- Disponibilidad de agua (no concesionada);

- En caso de que existan concesiones del agua, se pueden calcular sus remanentes disponibles, cantidad y temporalidad a fin de conocer el volumen 


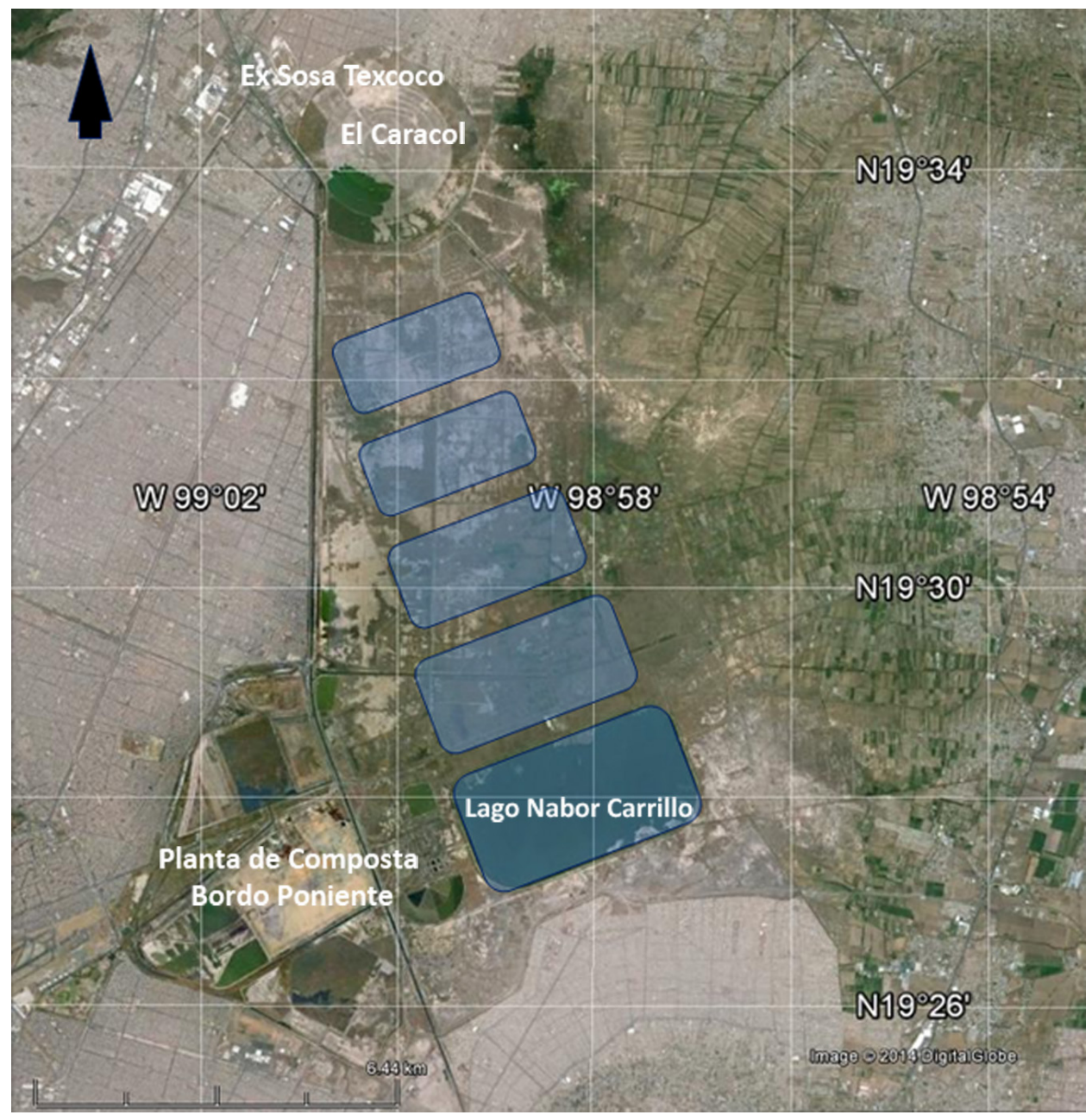

Figura 6. Sistema de lagos constituidos en forma de terrazas (azul). En amarillo, localización de fuentes de energía solar. Elaboración de los autores.

que puede ser aprovechado y con ello planear la ejecución de las obras hidráulicas;

- En caso de que el volumen disponible no sea suficiente, se podría establecer una programación de obra, en la cual se aproveche la temporada de lluvias, para que con los excedentes procedentes de esta agua, se puedan llenar los vasos que se proponen crear solicitando a la autoridad que determine los volúmenes excedentes que se podrían aprovechar por esta causa; $y$

- Los permisos y concesiones, en caso de que se requiera perforar un acuífero que se considere vedado.

\subsubsection{Balance Energético en la ZFLT}

Como requisito el sistema lacustre deberá ser autosuficiente energéticamente. La energía eléctrica para el bombeo, filtración y control del sistema se obtendría in situ ya sea por celdas fotovoltaicas u otro sistema de energías renovables que se decida operar, como pueden ser las fotovoltaicas flotantes (Patel, 2011). La filtración del agua podría estar asociada al sistema solar basado en el Sistema de Fresnel, como se describe en la propuesta de "Generación de electricidad y calor con energía solar".

Un ejemplo conservador para lograr un sistema de autoabastecimiento es: Con una eficiencia de paneles fotovoltaicos del $10 \%$ y suponiendo energía solar incidente de $6.3 \mathrm{~kW} \mathrm{~d} / \mathrm{m}^{2}$, con una superficie igual al del LNC de 904 ha se podrían generar $6 \mathrm{MWe}$ al día para sostener el sistema (incluyendo aeración mecánica). El excedente de energía eléctrica se exportaría a la Comisión Federal de Electricidad para consumo regional cercano.

La superficie generadora de electricidad y energía se construiría en una zona exclusiva para ello en la región suroeste de la ZFLT, o bien, usando estructuras ligeras para sostener paneles fotovoltaicos (PV's) o de Fresnel sobre las zonas lacustres. Cabe recordar que los PV's mantienen una mejor eficiencia de operación a temperaturas bajas, por lo que su cercanía a cuerpos de agua favorece esta situación. Esto se podría probar durante las etapas experimentales. La operación estaría sujeta a la insolación (días y horas con cielos claros luminosos), para no requerir de sistemas de almacenamiento de energía. 


\subsubsection{Beneficios ambientales}

En la Figura 4 se muestran los resultados de una modelación computacional de un episodio de emisión de PM proveniente de la ZFLT y las concentraciones resultantes con la situación actual y con un lago virtual en la región de Texcoco. Esta figura muestra que subsisten otras fuentes provenientes de zonas agrícolas al norte y sur-este de la ZMVM, pero que las emisiones de la zona de Texcoco desaparecen con un lago virtual. De manera similar en la Figura 7 se muestra una modelación computacional con resolución de $1 \mathrm{~km}$ con un lago virtual y sin éste en la ZFLT (López-Espinoza y Zavala-Hidalgo, comunicación personal). Se aprecia que localmente la presencia de la zona lacustre aumenta la velocidad del viento superficial y la temperatura disminuye durante el día. Se concluye que la creación de zonas lacustres abatiría la fuente de PM proveniente de Texcoco y en las zonas aledañas se incrementaría la ventilación reduciendo las concentraciones de la contaminación atmosférica y se modularía la temperatura. Esto confirma lo descrito en Jazcilevich et al. (2000, 2002).

Si a través del sistema circulatorio se logra obtener alta calidad de agua, ésta podría ser utilizada para los sistemas de bombeo de recuperación del acuífero que se localizan cerca. Para esto se podría usar la energía eléctrica generada en el lugar. El bombeo se programaría preferentemente antes del comienzo de la temporada de lluvia, destinando el agua de algunas terrazas para este fin dejando listo el sistema para recibir la precipitación pluvial. También, en las terrazas con

a)

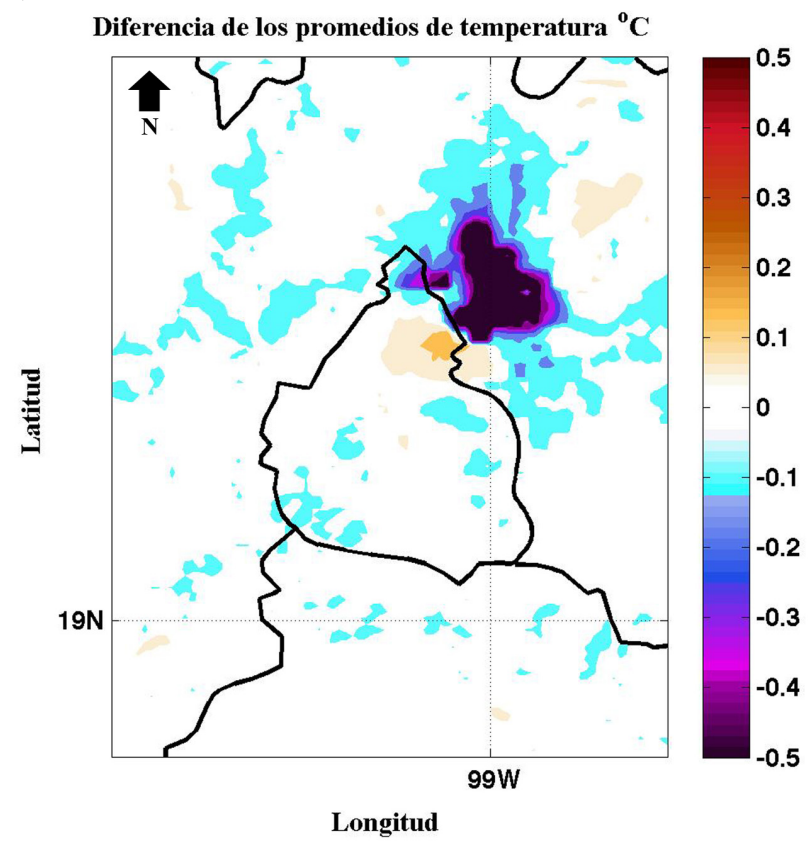

agua de alta calidad, se podría lograr el objetivo original del LNC y usarse para actividades recreativas de remo, canotaje y piscicultura.

\subsubsection{Desarrollo de la fase experimental.}

Para pasar de la fase conceptual y sustentar técnicamente la propuesta, se plantea una fase experimental para lograr las siguientes metas:

- Mediante el uso de modelos meteorológicos complejos y de calidad del aire, obtener la superficie crítica y la forma óptima de sistema lacustre para maximizar la ventilación.

- Obtener en campo los factores de emisión de aeropartículas de zonas desnudas y con vegetación.

- Obtener flujos de energía y materia de zonas lacustres ya existentes.

- Construcción de un sistema piloto de distribución y tratamiento de agua usando energías renovables.

- Obtener un modelo sistémico para el control y operación del sistema lacustre.

\subsection{Manejo de residuos sólidos orgánicos en la ZFLT}

La ZFLT cuenta con la infraestructura para el manejo de residuos sólidos orgánicos, como la planta de composta y los tiraderos del Bordo Poniente. Esto posibilita la utilización de residuos sólidos urbanos para la generación de electricidad y calor. Es posible también utilizar los residuos sólidos urbanos ya dispuestos en el relleno sanitario mediante b)

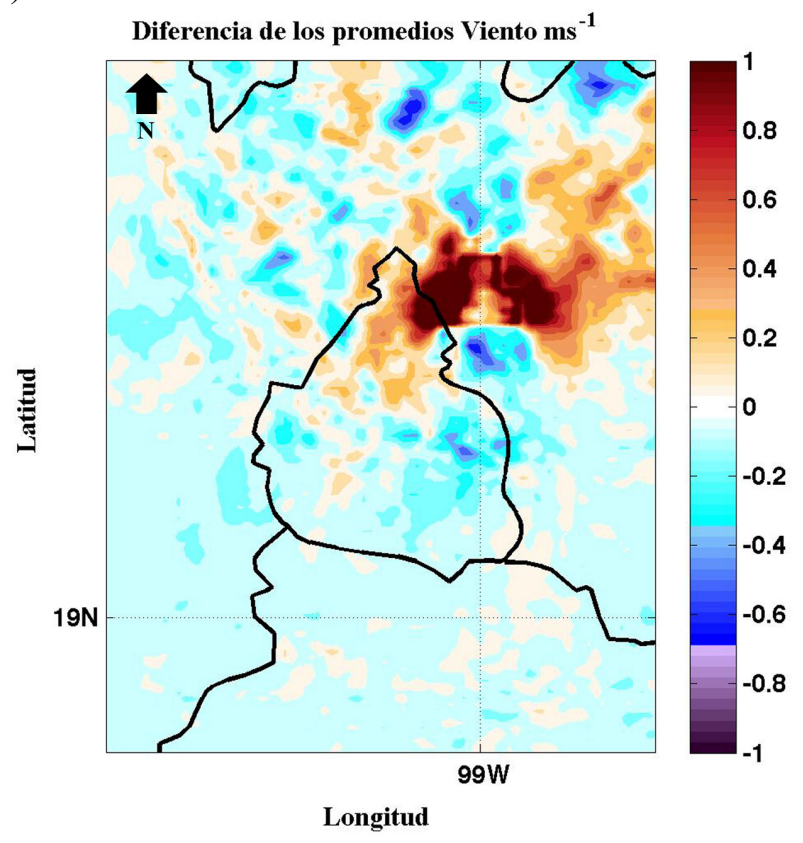

Figura 7. Modelación usando WRF con resolución horizontal de $1 \mathrm{~km}$, promedios con y sin lago durante 144 horas a partir de las 00:00 del 26 de marzo, 2011. En (a) diferencia de los promedios de velocidad del viento. Nótese el aumento de la velocidad debido a la presencia de la zona lacustre. En (b) diferencia de promedios de temperatura. Tomado de: López-Espinoza y Zavala-Hidalgo, comunicación personal. Elaboración de: López-Espinoza y Zavala-Hidalgo. 
tecnologías térmicas de manejo. Entre éstas se tienen la incineración, la pirólisis y la gasificación por lo que se estima un potencial energético pasivo importante en el sitio.

La gasificación de biomasa o residuos sólidos orgánicos urbanos consiste en generar un gas formado de monóxido de carbono, hidrógeno y bióxido de carbono, llamado gas sintético o syngas. Esto se logra mediante una reacción a altas temperaturas $\left(>700{ }^{\circ} \mathrm{C}\right)$, sin generar combustión de la sustancia carbonácea extraída de los residuos sólidos. El control se logra regulando la concentración de oxígeno y/o vapor. Este gas sintético se puede usar en procesos de combustión a altas temperaturas y, por lo tanto, con mayores eficiencias. Por esta razón se utiliza este proceso para generar electricidad en desarrollos industriales. Desde hace más de 30 años se ha incrementado el interés sobre este tema, siendo Estados Unidos uno de los principales promotores de dicho proceso (Leung et al., 2004; Ruiz et al., 2013a, 2013b). Esto se constata usando los datos de publicaciones en el área a partir de la base Scopus (2014).

Gracias a la presencia de tiraderos y de la planta de composta, existe la disponibilidad de materia prima para aprovechar su contenido energético mediante gasificación. Estos residuos que se generan continuamente, se verían ahora como un producto aprovechable para un ciclo productivo de energía, más que desechos que requieren de confinamiento. El calor, la energía mecánica y la electricidad generados, serían insumos para las otras opciones, ya sea para el tratamiento de agua, o bien, para el manejo de sistemas de bombeo y control. En el Instituto de Ingeniería de la UNAM está en operación el Laboratorio de Gasificación de Biomasa, por lo que ya se tiene experiencia en la materia.

\subsubsection{Beneficios ambientales en la ZFLT}

La mayor ventaja de esta opción sería aprovechar la transformación de residuos sólidos orgánicos producidos por la zona metropolitana donde ya se lleva a cabo la separación de los residuos sólidos, sanear suelos ahora ocupados por estos residuos, eliminando fauna nociva y malos olores. Darle valor a los residuos sólidos orgánicos desincentivaría la quema de éstos a cielo abierto y se podría generar biocarbón para abono, el cual facilitaría la rehabilitación de una cobertura vegetal, como ya se describió anteriormente.

\subsubsection{Viabilidad técnica y legal}

Dado que La Ley General para la Prevención y Gestión Integral de los Residuos, prohíbe el tratamiento térmico de los residuos sólidos orgánicos, la viabilidad legal de esta propuesta depende de los siguientes criterios:

- Que el proyecto no sea enfocado hacia un tratamiento térmico de residuos sólidos urbanos, sino como un tratamiento que promueve la valorización de residuos de manejo especial, mediante la generación de energía a partir de biomasa, para lo que se deben incorporar residuos de manejo especial agropecuarios y forestales (en particular los procedentes de podas de parques y jardines y los orgánicos procedentes de rastros), siendo la fracción orgánica de los sólidos urbanos, un insumo menor, para que no sea considerado un tratamiento térmico de estos residuos;

- La gasificación podría considerarse como un proceso que controla la generación de malos olores y lixiviados que se generan como resultado del proceso de compostaje, no como un fin en sí mismo; de esta manera se evita el supuesto de tratamiento térmico de residuos sólidos urbanos (prohibido por ley);

- Independientemente del tipo de tecnología, la definición del organismo responsable es fundamental, considerando las recientes reformas en materia energética, ya que de ello dependerá el tipo de regulación que aplicará en materia energética.

\subsubsection{Desarrollo de la fase experimental}

Se construirá en la ZFLT una planta de gasificación piloto de $1 \mathrm{MWe}$ de electricidad con un costo aproximado de US\$ 800000. Se obtendrían datos de consumo de residuos y costos asociados como su transporte, calidad y disponibilidad. Se procedería entonces a determinar el escalamiento de la planta, localización final y acoplamiento, conforme al desarrollo de las otras opciones.

\subsection{Generación de electricidad y calor con energía solar}

En la ZFLT existe una importante disponibilidad de energía renovable gracias a las dimensiones de su superficie $\left(60 \mathrm{~km}^{2}\right)$. Esto hace posible explotar en esta zona la energía solar para la generación de electricidad y calor con tecnología comercial ya disponible.

Gracias al desarrollo tecnológico en los últimos años, los sistemas fotovoltaicos y térmicos solares son cada vez más eficientes, por lo que muchos de ellos compiten en los mercados comerciales (Tabla 2) (Chow et al., 2012; Lueken et al., 2012; Desideri y Campana, 2014; Parastegari et al., 2015).

Los sistemas fotovoltaicos generan electricidad convirtiendo la radiación solar en corriente eléctrica directa. Esto sucede cuando los electrones en la banda de valencia del material fotovoltaico (como silicio) son excitados por la luz y se separan de su átomo pudiendo formar una corriente eléctrica. La primera aplicación fue para generar electricidad en los satélites espaciales. Actualmente la eficiencia de paneles solares comerciales es de alrededor del $14 \%$.

$\mathrm{Si}$ la radiación solar se concentra en una porción del material fotovoltaico mediante una lupa, como en los sistemas Fresnel, se incrementa la producción de electricidad, aunque aumenta el calor, bajando la eficiencia del sistema. Para contrarrestar esto, es necesario un sistema de enfriamiento, o bien, conjuntar el sistema Fresnel para utilizar el calor que genera para temperar un invernadero, como recientemente se ha hecho en varias partes del 
mundo (Sonneveld et al., 2011; Pérez-Alonso et al., 2012; Lamnatou y Chemisana, 2013; Cossu et al., 2014) (Figura 8).

\subsubsection{Beneficios ambientales}

Dependiendo de la extensión en superficie del sistema fotovoltaico se podrían satisfacer, en forma importante, los requerimientos de electricidad de la ZMVM y completamente las de otras propuestas. Se utilizaría la electricidad localmente y durante el día, para prescindir de sistemas de almacenamiento. La venta de energía ayudaría a recuperar la inversión.

La combinación de sistemas Fresnel e invernaderos generaría electricidad y agricultura de alto valor agregado, junto a un mercado importante como la ZMVM, donde se concentra la mayor actividad económica del país.

\subsubsection{Viabilidad técnica y legal}

Se requiere establecer institucionalmente al organismo responsable de generar y distribuir la energía. En función de su naturaleza, la fuente de generación que se defina, cantidad de energía generada y los usos para los que se disponga esa energía, se formará su marco legal específico.

Vale la pena agregar que con las iniciativas presentadas por el ejecutivo federal al Congreso de la Unión, se contará con un marco mucho más amplio de acción para este tipo de iniciativas, pudiendo además ser sujeto de instrumentos de fomento por parte del Estado Mexicano, ya que permitiría cumplir con la meta de generación de renovables a 2024 prevista en la Ley General de Cambio Climático.

\subsubsection{Desarrollo de la fase experimental}

Se mediría la calidad de la energía solar, radiación directa, radiación difusa, albedo, así como niveles de insolación y turbiedad en la ZFLT. Para valorar y evaluar el sistema Fresnel-invernadero se crearía una granja en la zona. Para proceder a su escalamiento, se determinarían sus insumos, requerimientos y producción. Será importante establecer la introducción al mercado de sus productos agrícolas.

Para el caso de celdas fotovoltaicas, se construiría una planta piloto de $10 \mathrm{MWe}$ de generación de electricidad en dos años, con un costo de 2 millones de dólares. Una vez que se realicen los estudios de costo-beneficio, se evalúe y determine su utilidad y posibilidades de interconexión con usuarios diurnos, se decidiría el nivel de escalamiento.

\section{5. ¿Y la ampliación del Aeropuerto de la Ciudad de México?}

El Sistema Aeroportuario Nacional se compone de 60 aeropuertos de los cuales, 17 concentran el $86 \%$ del tránsito de pasajeros y el $96 \%$ de la carga aeroportuaria (PND, 20132018). Esto denota la disparidad en el uso de los aeropuertos, pues mientras algunos de ellos son subutilizados otros se encuentran saturados, como el AICM. Por otra parte, la calidad de la infraestructura aeroportuaria en la región centro es baja, limitando la conectividad del país así como su crecimiento y productividad (PND, 2013-2018).

En el caso del AICM su vida útil está limitada por su capacidad operacional (Torres-Guadarrama, 2011) lo que justifica la construcción de un nuevo aeropuerto que lo sustituya. El AICM puede seguir operando, siempre y cuando la infraestructura se amplíe y mejore (TorresGuadarrama, 2011). Esta necesidad de ampliación es bien conocida desde hace algunas décadas, sin embargo, por diversas circunstancias, su realización no ha sido posible (Meléndez y Buendía, 2007). En 1985, el gobierno de Miguel de la Madrid tomó la decisión de ampliar el aeropuerto capitalino en el exlago de Texcoco, la cual fue detenida debido al proceso de recuperación ambiental al que estaba sujeta la zona.

En el 2001, durante la administración de Vicente Fox, se decidió construir un nuevo aeropuerto localizado parcialmente en la ZFLT y en Atenco, Estado de México. Entre las ventajas que ofrecía este proyecto, estaba su cercanía al centroide de la demanda, localizada en la Fuente de Petróleos en Periférico y Paseo de la Reforma. En comparación con otras posibles localidades como Tizayuca, Hidalgo, el estudio ambiental realizado por la UNAM, no concluyó que hubiera comparativamente ventajas o desventajas importantes, aunque la localización en Tizayuca hubiera podido ofrecer ventajas en cuanto a calidad del aire bajo ciertas condiciones de crecimiento urbano (PUMA, 2001). Este proyecto no se llevó a cabo debido a las protestas sociales que se suscitaron, que son de conocimiento público.

Sin embargo, en el 2013 comenzó nuevamente a circular información sobre la ampliación del AICM, así como la definición del posible lugar: La Zona Federal del exlago de Texcoco (El Economista, 2013b).

\section{Comentarios y opiniones sobre la ampliación del Aeropuerto de la Ciudad de México en la Zona Federal del Lago de Texcoco}

Este proyecto implicará obras de gestión y acondicionamiento hidrológico, equipamiento e infraestructura urbana (conectividad y accesibilidad vial) y restauración ecológica (El Economista, 2013a). Se contempla que este nuevo aeropuerto deberá contar con al menos dos pistas paralelas, una terminal y la capacidad para mover un mínimo de 50 millones de pasajeros al año (El Financiero, 2013).

Sin embargo, esta propuesta se basa en las necesidades de desarrollo urbano, problemática ambiental y la tecnología existente de principios del Siglo XXI. En esos años, empezaba la discusión internacional acerca de la existencia y efectos del Cambio Climático Global, aún no existían los cuestionamientos acerca de la disponibilidad de energía fósil y no se contaba con los avances tecnológicos en generación 
a)

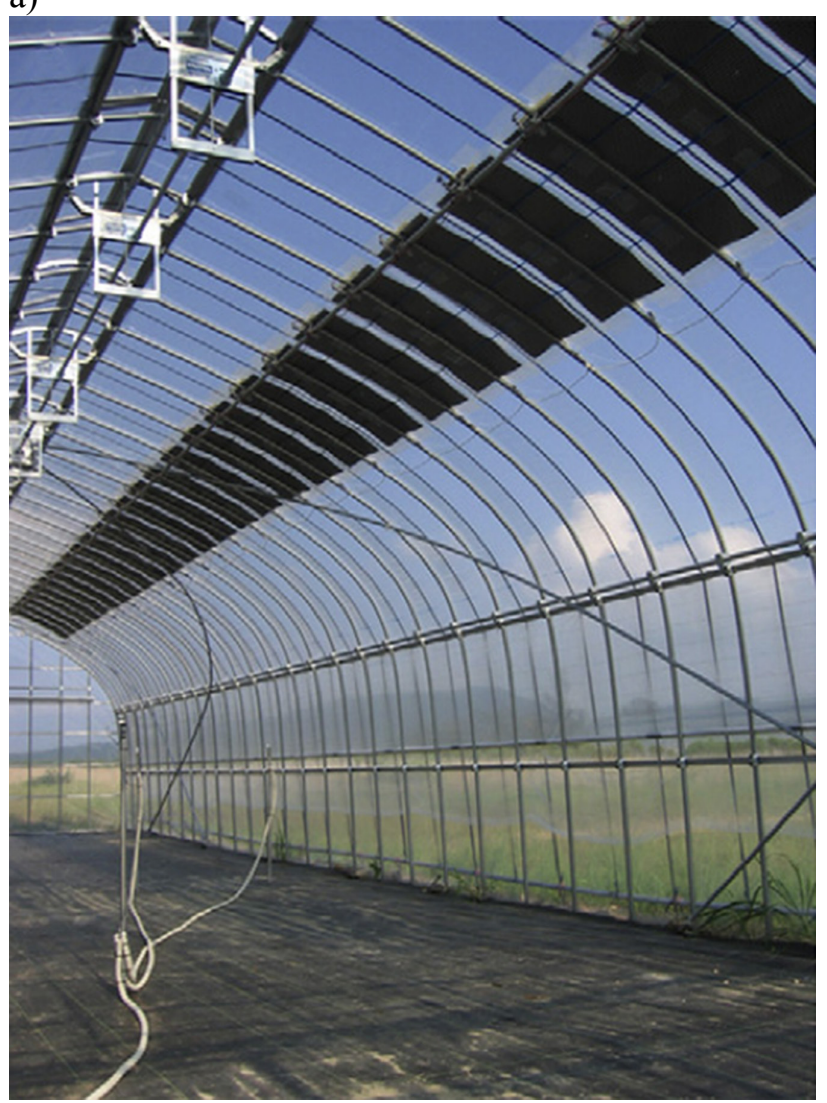

b)

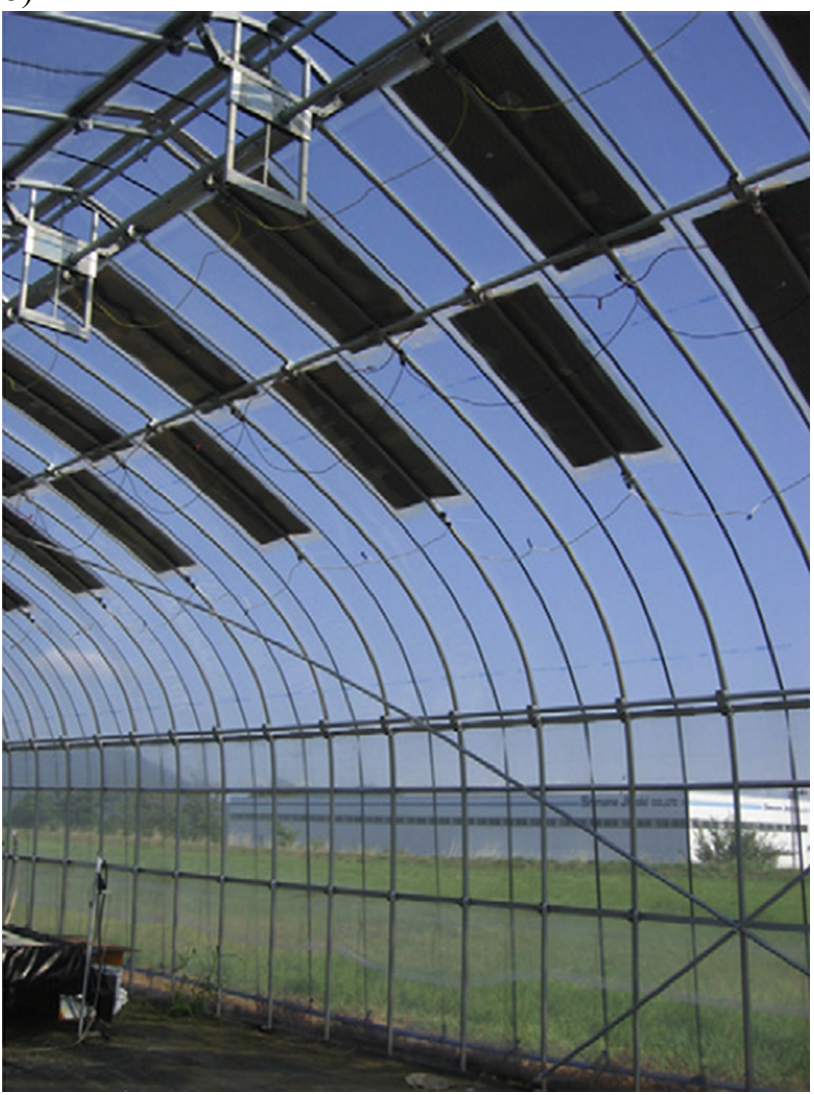

c)

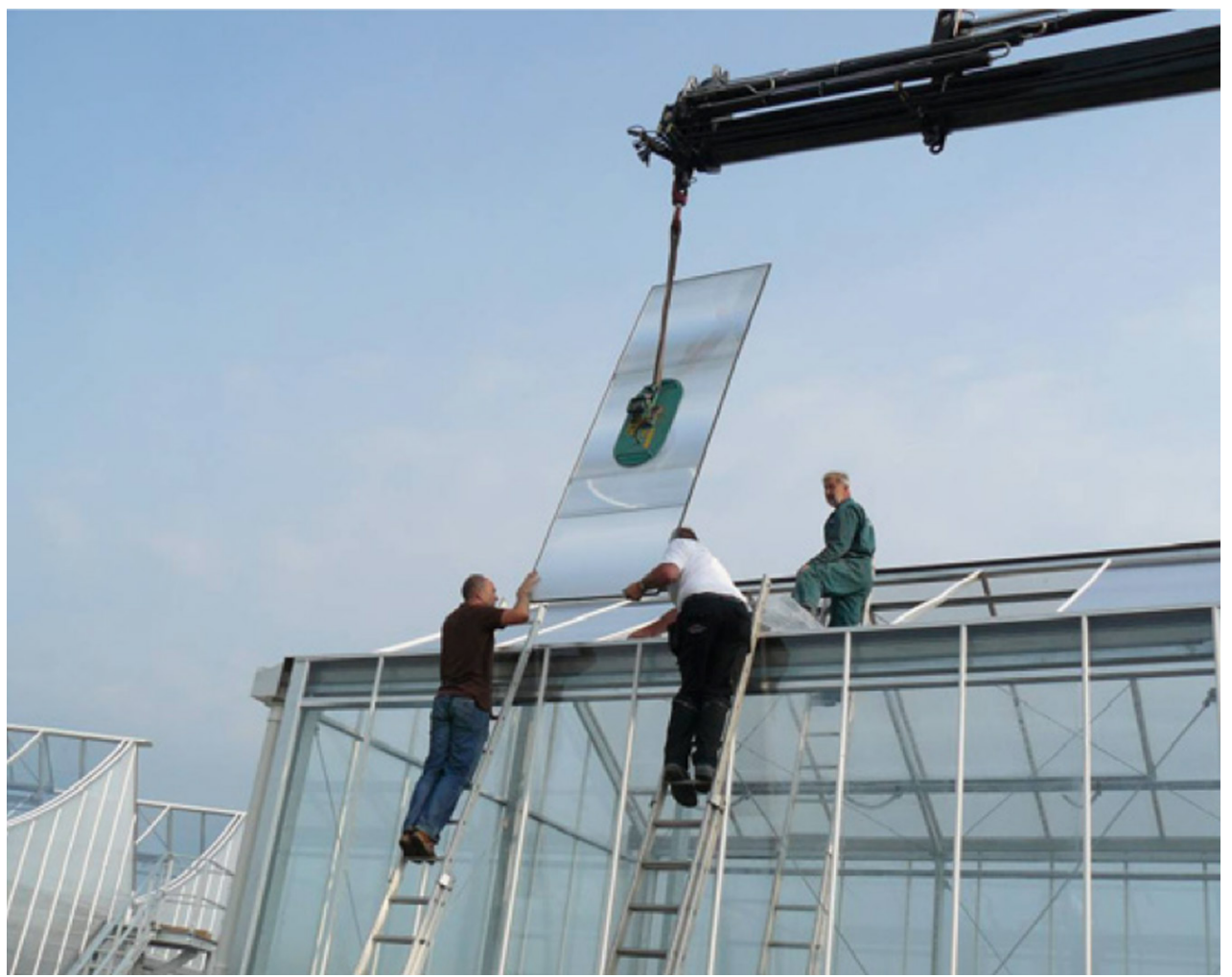

Figura 8. Las lupas tipo Fresnel dirigen los rayos solares en función de la orientación del sol y de la época del año, esto permite el paso de luz y la generación de electricidad en un invernadero. En (a y b) disposición de paneles solares dentro del techo de un invernadero. Los módulos solares fueron arreglados en una línea recta o en forma de tablero. En (c) montaje de un panel fotovoltaico de tipo Fresnel sobre un invernadero. Tomado de: Sonneveld et al. (2011) y Yano et al. (2010). 
de energía renovable a través de diferentes fuentes como la solar y aprovechamiento de residuos sólidos orgánicos urbanos con los que ahora se cuentan. En nuestra opinión, esta nueva situación cambia la valoración económica y ambiental de la ZFLT. En la actualidad existe la oportunidad de generar electricidad renovable localmente, remediar suelos para la producción alimentaria de alto valor agregado y crear zonas lacustres con fines recreativos y de regulación hidráulica y térmica. Nos encontramos en la coyuntura para desarrollar zonas de oportunidad económica sostenibles y amigables con el ambiente que no existían entonces.

Con la información disponible acerca de la ampliación del aeropuerto en la ZFLT no se puede realizar una comparación económica-ambiental formal con nuestras propuestas. Sin embargo, la construcción de una obra civil estratégica, de gran magnitud y de uso continuo e intensivo en una zona con suelos cuya mecánica y composición química es especialmente difícil, conlleva a altos costos de construcción y mantenimiento. Esta situación pondría al aeropuerto en desventaja competitiva con otros aeropuertos internacionales que necesitarán menores presupuestos de operación y mantenimiento.

\subsection{Viabilidad técnica y legal}

Al no conocer los detalles técnicos de la obra, tan sólo nos atrevemos a realizar las siguientes conjeturas acerca de su viabilidad técnico - económica:

1. Los costos de construcción de pistas, almacenes, obras de conectividad, accesibilidad vial y demás infraestructura necesaria para la operación del nuevo aeropuerto serán altos debido a los suelos altamente salinos, con movimientos de la sal y hundimientos en la ZFLT, como se mencionó en la sección de Rehabilitación de suelos y vegetación.

2. Los costos de mantenimiento de las construcciones serán altos, quizás cerca del $10 \%$ del costo inicial, por las mismas razones antes mencionadas.

3. Los costos de operación serán elevados, entre otras razones, para mitigar partículas suspendidas por las áreas de suelo erosionable aledañas a las pistas y prevención de inundaciones.

4. Los costos para la disponibilidad de agua serán altos para el abastecimiento de los servicios del aeropuerto y el cumplimiento de la normatividad respectiva.

5. Con la información que se cuenta, el Nuevo Aeropuerto Internacional de la Ciudad de México (NAICM) está concebido como una obra pública para la Ciudad de México, cuando debería de estar concebida en el contexto de la megalópolis, es decir, las zonas urbanas de Toluca, Cuernavaca, PueblaApizaco, Pachuca y la ZMVM (GODF, 2003). Ejemplo de ello es que no incluye terminales para la conexión con transporte público de la Región Central de México (Grupo Milenio, 2014; SCT,
2014).

Con respecto a la viabilidad legal consideramos que:

- La ZFLT, es propiedad de la Federación y la Comisión Nacional del Agua (CONAGUA) está a cargo de su manejo, por lo que el posible conflicto asociado con la tenencia de la tierra es nulo. Sin embargo, el uso de suelo, deberá ser compatible con el urbano. Por otra parte, los terrenos adyacentes, tienen una tenencia mixta, siendo ésta la principal limitante al proyecto.

- Dado que es una obra, que de acuerdo con la Ley General del Equilibrio Ecológico y la Protección al Ambiente, requiere de una Evaluación de Impacto Ambiental previa a su construcción, será necesario que este proceso considere que la consulta pública pudiera incluir una serie de actores que se manifestarán en contra del proyecto, por lo que el promotor, deberá contar con un equipo sólido de expertos para contrarrestar las objeciones técnicas al proyecto. Lo anterior sin considerar la posibilidad de que se presenten manifestaciones sociales contrarias al mismo, las cuales requerirán de un manejo de conflictos eficiente.

- Los permisos de construcción corresponderán a la autoridad municipal, la cual, por el momento, no representa un obstáculo a la construcción del mismo.

\subsection{Compatibilidad con algunas de nuestras propuestas}

La ampliación del AICM en la ZFLT es compatible con:

- La utilización de la energía solar con sistemas fotovoltaicos y fototérmicos para generación de electricidad pero es necesario establecer límites, esto es, cumplir la regulación aeroportuaria. Una limitante podría ser el efecto reflector de la infraestructura de energía solar.

- La rehabilitación de zonas lacustres, del suelo y de la vegetación en zonas libres de infraestructura aeroportuaria.

\subsection{Beneficios y costos ambientales}

Con el sellado del suelo por medio de la construcción de la infraestructura aeroportuaria habría una mayor capacidad operacional del actual AICM además de creación de empleos. También habría una disminución de la erosión eólica y por lo tanto de tolvaneras, pero a un alto costo de operación y mantenimiento. Entre los costos ambientales de esta opción tenemos que la ampliación del AICM conllevará a la urbanización de la zona noreste y como consecuencia la emisión de PMs y gases criterio por el uso de combustibles fósiles, lo cual podría tener un efecto negativo en la calidad del aire del valle de México, como se muestra en Jazcilevich et al. (2003a, 2003b). 


\section{Conclusiones}

La utilización de la gran extensión territorial de ZFLT es inaplazable y se encuentra en una encrucijada: se le convierte en un aeropuerto cercano a la demanda de transporte, o se le aprovecha para mitigar problemas de energía, gestión hidráulica y de calidad del aire y con ello promover el desarrollo económico y social de la región central del país.

La tecnología de energías renovables que se ha desarrollado debido a la crisis energética mundial, y la preocupación generada por la agudización de los problemas locales acerca de uso de suelo, calidad del aire y gestión hidráulica, hace que reconsideremos cuales son las mejores opciones para el futuro sustentable y sostenible de la ZFLT. Las soluciones tecnológicas que se incluyen en las propuestas de este documento, sacan ventaja de los desarrollos tecnológicos en fuentes renovables y el aprovechamiento de residuos orgánicos sólidos para generar energía. Esta energía se usaría para aliviar problemas, algunos interrelacionados, como los hundimientos del suelo proveyendo energía para operar los pozos de recarga; operar bombas y plantas de tratamiento para resarcir la falta de agua; funcionamiento de invernaderos; riego para la rehabilitación de suelos y evitar así la emisión de material particulado proveniente de la ZFLT. Por eso todas las propuestas incluyen la generación de electricidad para autoabastecimiento o para exportación. Las tecnologías generadoras de electricidad están disponibles en el mercado y se tiene experiencia local en ellas. Otras, como la de rehabilitación de suelos y vegetación, así como la de regeneración de zonas lacustres, habrán de desarrollarse o ampliarse. Se plantea la posibilidad de generar agua de buena calidad que permita el bombeo y recarga de acuíferos, así como el riego de hortalizas.

Las opciones tecnológicas propuestas en el presente trabajo son compatibles entre ellas, con cauce legal y aprovechan la circunstancia geográfica y ambiental de la ZFLT. También preparan el camino para considerar nuevas tecnologías de transporte y ahorro de energía del futuro. Durante una etapa experimental, todas las propuestas se pueden probar antes de implementarse mediante proyectos piloto. Esto asegurará su viabilidad, su escalamiento y grado de interacción.

Creemos que todo lo anterior constituye un proyecto que potenciará las funciones actuales del suelo y de manera integral involucrará a las comunidades aledañas a la ZFLT de forma constructiva, productiva y sostenible, además de mitigar problemas ambientales y de gestión hidráulica, es decir, convertirá estos retos en oportunidades.

La propuesta de localizar el NAICM en la ZFLT no es una solución integral con respecto a la problemática de energía, transporte, calidad del aire, manejo hidráulico, residuos y suelos que enfrenta la ZMVM. Tan sólo trata de aliviar el rezago de transporte aéreo, específicamente de la Ciudad de México, sin tomar en cuenta los problemas de la megalópolis.

¿Cuál es la opción más conveniente a largo plazo para la megalópolis: un aeropuerto en la ZFLT o considerar las propuestas aquí descritas?

\section{Agradecimientos}

Este trabajo fue financiado por Climate Works Foundation. Agradecemos la ayuda de la Dra. Telma Gloria Castro Romero, Directora del Centro de Ciencias de la Atmósfera y de la M. en C. Mireya Imaz Gispert, Coordinadora del Programa Universitario del Medio Ambiente (PUMA) para llevar a cabo los talleres. A la Consultora Geoda, Geosistemas y Desarrollo Ambiental S.C. por las aportaciones a este informe. Al Dr. Emmanuel Díaz-Nigenda y a la Dra. Erika López-Espinoza por la elaboración de imágenes. Al Dr. Oscar Arnoldo Escolero y al Dr. Gabriel Auvinet Guichard por sus contribuciones y a los ponentes y asistentes a los talleres y mesas de trabajo.

\section{Referencias}

Aguilar-Virgen, Q., Taboada-González, P., Ojeda-Benítez, S., 2014, Analysis of the feasibility of the recovery of landfill gas: a case study of Mexico: Journal of Cleaner Production, 79, 53-60.

Alcocer-Durand, J., Escobar-Briones, E., 1992, The aquatic biota of the now extinct lacustrine complex of the Mexico basin: Freshwater Forus, 2(3), 171-183.

Babaee, S., Nagpure, A.S., DeCarolis, J.F., 2014, How much do electric drive vehicles matter to future us emissions: Environmental Science and Technology, 48(3), 1382-1390.

Brady, N.C., Weil, R.R., 2002, The nature and properties of soils: U.S., Prentice Hall, 960 p.

Cámara Mexicana de la Industria de la Construcción (CMIC), 2013, Infraestructura eléctrica, Agenda e incidencia de la industria de la construcción en México (en línea): México, D.F., Cámara Mexicana de la Industria de la Construcción, disponible en <http:/www. cmic.org/agendaindustria/AGENDA\%20ELECTRICIDAD.pdf>, consultado 29 de marzo de 2014.

Carrera-Hernández, J.J., Gaskin, S.J., 2009, Water management in the Basin of Mexico: current state and alternative scenarios: Hydrogeology Journal, 17, 1483-1494.

Chow, T.T., Tiwari, G.N., Menezo, C., 2012, Hybrid Solar: A Review on photovoltaic and thermal power integration: International Journal of Photoenergy, 2012, 1-17.

Comisión Nacional del Agua y Universidad Autónoma Chapingo (CONAGUA y UAch), 2004, Plan de Consolidación del Rescate Hidroecológico de la Zona Federal del ex Lago de Texcoco, Segunda parte: Evaluación y diagnóstico de acciones 1971-2003: México, D.F., Gerencia Regional de Aguas del Valle de México y Sistema Cutzamala Gerencia de Programación, 173 p.

Comisión Nacional del Agua (CONAGUA), 2010, Relleno Sanitario BORDO PONIENTE, IV Etapa, Firma del convenio de coordinación para el cierre y clausura definitiva (en línea): México, D.F., Comisión Nacional del Agua, disponible en <http://www.conagua.gob.mx/ CONAGUA07/Contenido/Documentos/CONVENIO\%20DE\%20 COORDINACION\%20CIERRE \%20B.\%20PONIENTE $\% 20 I V \% 20$ ETAPA.pdf $>$, consultado 16 de abril de 2014.

Comisión Nacional del Agua (CONAGUA), 2011, Estadísticas del agua en México: México, D.F., Comisión Nacional del Agua, 181 p.

Comisión Nacional del Agua (CONAGUA), 2012, Acciones de infraestructura de drenaje y abastecimiento de agua en el Valle de 
México 2007-2012, Coordinación General de Proyectos Especiales de Abastecimiento de Agua Potable y Saneamiento del Valle de México: México, D.F., Comisión Nacional del Agua, 119 p.

Cossu, M., Murgia, L., Ledda, L., Deligios, P.A., Sirigu, A., Chessa, F., Pazzona, A., 2014, Solar radiation distribution inside a greenhouse with south-oriented photovoltaic roofs and effects on crop productivity: Applied Energy, 133, 89-100.

Cruickshank, G., 2007, Proyecto lago de Texcoco: rescate hidroecológico: México, D.F., Comisión Nacional del Agua-SEMARNAT, 140 p.

Delucchi, M.A, Yang, C., Burke, A.F., Ogden, J.M., Kurani, K., Kessler, J., Sperling, D., 2014, An assessment of electric vehicles: technology, infrastructure requirements, greenhouse-gas emissions, petroleum use, material use, lifetime cost, consumer acceptance and policy initiatives: Philosophical Transactions of the Royal Society A, 372, 1-27.

Desideri, U., Campana, P.E., 2014, Analysis and comparison between a concentrating solar and a photovoltaic power plant: Applied Energy, $113,422-433$

Díaz-Nigenda, E., Tatarko, J., Jazcilevich, A.D., García, A.R., Caetano, E., Ruíz-Suárez, L.G., 2010, A modeling study of Aeolian erosion enhanced by surface wind confluences over Mexico City: Aeolian Research, 2, 143-157.

Ducks Unlimited de México, A.C. (DUMAC), 2005, Programa de conservación y manejo para las aves playeras en el Lago de Texcoco, Estado de México (en línea): N.L. México, Ducks Unlimited de México, A.C., disponible en $<$ http://www.dumac.org/dumac/habitat/ esp/pdf/Informe-Final-Texcoco.pdf $>$, consultado 7 de octubre de 2014.

El Economista, 2013a, El DF, socio del nuevo aeropuerto en Texcoco, artículo (en línea): El Economista, actualizado 26 de julio de 2013, disponible en $<$ http://eleconomista.com.mx/columnas/columnaespecial-empresas/2013/07/26/df-socio-nuevo-aeropuerto-texcoco>, consultado 5 de marzo de 2014.

El Economista, 2013b, Nuevo aeropuerto será en Texcoco, artículo (en línea): El Economista, actualizado 2 octubre de 2013, disponible en $<$ http://eleconomista.com.mx/foro-economico/2013/10/02/ nuevo-aeropuerto-sera-texcoco $>$, consultado 4 de marzo de 2014.

E1 Financiero, 2013, Expertos 'aterrizan' el nuevo aeropuerto, artículo (en línea): El Financiero, actualizado18 octubre de 2013, disponible en $<$ http://www.elfinanciero.com.mx/archivo/expertos-aterrizan-elnuevo-aeropuerto.html>, consultado 4 de marzo de 2014.

Energy Information Administration (EIA), 2012, Most states have Renewable Portfolio Standards (en línea): U.S., Energy Information Administration, actualizado 03 de febrero de 2012, disponible en <http://www.eia.gov/todayinenergy/detail.cfm?id=4850>, consultado 6 de abril de 2014.

Energy Information Administration (EIA), 2014, Levelized Cost and Levelized Avoided Cost of New Generation Resources in the Annual Energy Outlook 2014 (en línea): U.S., Energy Information Administration, actualizado 03 de febrero de 2012, disponible en $<\mathrm{http}: / \mathrm{www}$. eia.gov/todayinenergy/detail.cfm?id=4850>, consultado 6 de abril de 2014.

Erkens, G., Bucx, T., Dam, R., de Lange, G., Lambert, J., 2014a, Sinking coastal cities (abstract), in European Geosciences Union General Assembly: Vienna, Austria, Geophysical Research Abstracts Vol. 16

Erkens, G., Dam, R., de Lange, G., Lambert, J., 2014b, Sinking coastal cities (abstract), in Nederlands Aardwetenschappelijk Congres (NAC12): Koningshof, Veldhoven.

Escobar, D.J., Jiménez, J.S., 2009, Urbanismo y sustentabilidad: estado actual del desarrollo urbano de la ZMVM: Revista Digital Universitaria [en línea], 10(7), disponible en: $<$ http://www.revista. unam.mx/vol.10/num7/art40/int40.htm $>$, consultado 8 de abril de 2014.

European Biochar Certificate (EBC), 2013, Guidelines for a Sustainable Production of Biochar, Version 4.5; Delinat Institut and Biochar Science Network: Ayent, Switzerland, European Biochar Foundation, disponible en $<$ http://www.european-biochar.org/biochar/media/ doc/1358641517626.pdf $>$, consultado 4 de agosto de 2014 .
Ezcurra, E., 1990, De las chinampas a la megalópolis: el medio ambiente en la Cuenca de México: México, Fondo de Cultura Económica, 119 p.

Fernández-Buces, N., 2006, Variabilidad espacial de la salinidad y su efecto en la vegetación en el ex Lago de Texcoco: implicaciones para su monitoreo por percepción remota: México D.F., Universidad Nacional Autónoma de México, tesis doctoral, 210 p.

Fernández-Buces, N., Siebe, C., Palacio, J.L., Cram, S., 2006, Mapping soil salinity using a combined spectral response index for bare soil and vegetation; a case study in the former lake Texcoco, Mexico: Journal of Arid Environments, 65, 644-667.

Fernández-Buces, N., Siebe, C., Palacio-Prieto, J.L., Webster, R., 2009, Mapping Soil Salinity from Sample Data and Remote Sensing in the Former Lake Texcoco, Central Mexico, in Metternicht, G., Zinck, J.A. (eds.), Remote Sensing of Soil Salinization Impact on Land Management: CRC Press, 291-304.

Fuel Economy, 2014, All-Electric Vehicles (en línea): U.S., Department of Energy, Energy Efficiency \& Renewable Energy, actualizado 25 de mayo de 2014, disponible en <http://www.fueleconomy.gov/feg/ evtech.shtml>, consultado 25 de mayo de 2014.

Fundación México Estados Unidos para la Ciencia (FUMEC), 2014, Sector Solar (en línea): México, D.F., Fundación México Estados Unidos para la Ciencia, disponible en $<\mathrm{http} / / /$ fumec.org/v6/htdocs/solar. pdf $>$, consultado 27 de mayo de 2014.

Gaceta Oficial del Distrito Federal (GODF), 2003, Decreto por el que se aprueba el Programa General de Desarrollo Urbano del Distrito Federal (en línea): México, D.F., Gobierno del Distrito Federal, disponible en $<$ http://www.aldf.gob.mx/archivo-2ec5c052a850fa2 a8491 ca817aee8439.pdf >, consultado 2 de octubre de 2014.

Gaceta Oficial del Distrito Federal (GODF), 2010, Acuerdo por el que se aprueba y expide el Programa de Gestión Integral de los Residuos Sólidos para el Distrito Federal (en línea): México, D.F., Gobierno del Distrito Federal, disponible en <http://www.sma.df.gob.mx/ rsolidos/03/local/03clave.pdf >, consultado 2 de junio de 2014 .

García-Escalante, J.S., García-Reynoso, J.A., Jazcilevich-Diamant, A., Ruiz-Suárez, L.G., 2014, The influence of the Tula, Hidalgo complex on the air quality of the Mexico City Metropolitan Area: Atmósfera, 27(2), 215-225.

Gobierno del Estado de Nuevo León, México (GENL), 2014, Planta BENLESA para generar energía eléctrica a través de los desechos (en línea): Gobierno del Estado de Nuevo León, México, disponible en $<$ http://www.nl.gob.mx/pics/pages/simeprode_bioenergia_base/ Benlesa.pdf $>$, consultado 1 de octubre de 2014.

Grupo Milenio, 2014, Proyectan 12 vías de acceso al aeropuerto, artículo (en línea): Grupo Milenio, actualizado 29 de septiembre de 2014, disponible en <http://www.milenio.com/politica/ acceso_aeropuerto-nuevo_aeropuerto-aeropuerto_df-aeropuerto mexico_0_367163292.html >, consultado 29 de septiembre de 2014 .

Iglesias-Piña, D., 2007, Costos económicos por la generación y manejo de residuos sólidos en el municipio de Toluca, Estado de México: Equilibrio Económico, 3(2), 131-148.

Ingenieros Civiles Asociados (ICA), 2011, Hidroeléctricas, (en línea): Ingenieros Civiles Asociados, disponible en $<$ http://www.ica.com. $\mathrm{mx} /$ central-hidroeléctrica-el-infiernillo.html $>$, consultado $18 \mathrm{de}$ marzo de 2014.

Instituto Nacional de Estadística y Geografía (INEGI), 2011a, Anuario de estadísticas por entidad federativa 2011: México. D.F., Instituto Nacional de Estadística y Geografía, 596 p.

Instituto Nacional de Estadística y Geografía (INEGI), 2011b, Cuaderno estadístico de la Zona Metropolitana del Valle de México 2011 (en línea): México. D.F., Instituto Nacional de Estadística y Geografía, disponible en $<$ http://www.inegi.org.mx/est/contenidos/espanol/ sistemas/cezm11/estatal/default.htm $>$, consultado 16 de abril de 2014.

International Biochar Initiative (IBI), 2012, Standardized Product Definition and Product Testing Guidelines for Biochar that is Used in Soil, International Biochar Initiative, disponible en $<\mathrm{http}$ :// www.biochar-international.org/sites/default/files/Guidelines_for_ Biochar_That_Is_Used_in_Soil_Final.pdf $>$, consultado 4 de agosto 
de 2014 .

Jazcilevich, A.D., Fuentes, V., Jáuregui, E., Luna, E., 2000, Simulated urban climate response to historical land use modification in the Basin of Mexico: Climatic Change, 44, 515-536.

Jazcilevich, A.D., García, A.R., Ruiz-Suárez, L.G., 2002, A modelling study of air pollution modulation through land-use change in the Valley of Mexico: Atmospheric Environment, 36, 2297-2307.

Jazcilevich, A.D., García, A.R., Ruiz-Suárez, L.G., 2003a, An air pollution modelling study using three surface coverings near the New International Airport for Mexico City: Journal of Air and Waste Management Association, 53, 1280-1287.

Jazcilevich, A.D., García, A.R., Ruiz-Suárez, L.G., Cruz-Núñez, X., Delgado, J.C., Tellez, C., Chias, L.B., 2003b, An air quality modelling study comparing two possible sites for the New International Airport for Mexico City: Journal of Air and Waste Management Association, 53, 366-378.

Jazcilevich, A.D, Grutter, M., García, A.R., Diego, U.A., Delgado, J., Suárez, M.L., Zuk, M., González, R.O., Lents, J., Davis, N., 2011, An evaluation of the hybrid car technology for the Mexico Mega City: Journal of Power Sources, 196, 5704-5718.

Kopp, D., Jäger, K. Succow, M., 1982, Naturräumliche Grundlagen der Landnutzung: Akademie Verlag, Berlin, 339 p.

Lamnatou, C., Chemisana, D., 2013, Solar radiation manipulations and their role in greenhouse claddings: Fresnel lenses, NIR- and UV-blocking materials: Renewable and Sustainable Energy Reviews, 18, 271-287.

Lehmann, J., 2007, Bio-Energy in the Black: Frontiers in Ecology and the Environment, 5, 381-387.

Leung, D.Y.C., Yin, X.L., Wu, C.Z., 2004, A review on the development and commercialization of biomass gasification technologies in China: Renewable and Sustainable Energy Reviews, 8, 565-580.

Lueken, C., Cohen, G.E., Apt, J., 2012, Costs of solar and wind power variability for reducing $\mathrm{CO}_{2}$ Emissions: Environmental Science \& Technology, 46, 9761-9767.

Maderey, L.E., Carrillo, J.J., 2005, El recurso agua en México: un análisis geográfico: México, D.F., Instituto de Geografía, UNAM, 128 p.

Maya, C., 2000, Modificación de la calidad físico-química y microbiológica de los efluentes de agua tratada por su embalse en el Lago Nabor Carrillo: México D.F., Universidad Nacional Autónoma de México, tesis de maestría, $137 \mathrm{p}$.

Meléndez, G.K.P., Buendía, M., 2007, El nuevo aeropuerto de la Ciudad de México en la zona oriente del Estado de México y sus desafíos: la resistencia civil de Atenco. ¡Tierras si aviones no!: reportaje: México D.F., Universidad Nacional Autónoma de México, tesis de licenciatura, $152 \mathrm{p}$.

Mendoza-Archundia, E., 2012, Caracterización hidrogeológica de la porción sureste de la planicie de Texcoco, México, para establecer sitos de recarga artificial al acuífero: México D.F., Universidad Nacional Autónoma de México, tesis de licenciatura, 160 p.

Mondragón-Rodríguez, R.D., 2013, Energía Solar y Cambio Climático en el Distrito Federal: México D.F., Universidad Nacional Autónoma de México, tesis de maestría, 215 p.

Moreno, P.S., Meixueiro, G., (coord.), 2007, El desarrollo metropolitano y la sustentabilidad de las ciudades: México, D.F., Centro de Estudios Sociales y de Opinión Pública Cámara de Diputados/LX Legislatura, $157 \mathrm{p}$.

Oswald, U., 2011, Aquatic systems and water security in the Metropolitan Valley of Mexico City: Current Opinion in Environmental Sustainability, 3, 497-505.

Ovando-Shelley, E., Ossa, A., Romo, M.P., 2007, The sinking of Mexico City: Its effects on soil properties and seismic response: Soil Dynamics and Earthquake Engineering, 27, 333-343.

Parastegari, M., Hooshmand, R., Khodabakhshian, A., Zare, A., 2015, Joint operation of wind farm, photovoltaic, pump-storage and energy storage devices in energy and reserve markets: Electrical Power and Energy Systems 64, 275-284.
Patel, S., 2011, Interest, funding buoys floating solar power plants: Power $155(5), 14$.

Pérez-Alonso, J., Pérez-García, M., Pasamontes-Romera, M., CallejónFerre, A.J., 2012, Performance analysis and neural modelling of a greenhouse integrated photovoltaic system: Renewable and Sustainable Energy Reviews, 16, 4675-4685.

Plan Nacional de Desarrollo (PND), 2013-2018, Gobierno de la República: México, Presidencia de la República, 183 p.

Programa Universitario de Medio Ambiente (PUMA), 2001, Evaluación comparativa de los sitios para la ubicación del Nuevo Aeropuerto Internacional de la Ciudad de México (NAICM) (Resumen ejecutivo): México, D.F., Proyecto SEMARNAT-UNAM.

Querol, X., Pey, J., Minguillón, M.C., Pérez, N., Alastuey, A., Viana, M., Moreno, T., Bernabé, R.M., Blanco, S., Cárdenas, B., Vega, E., Sosa, G., Escalona, S., Ruiz, H., Artíñano, B., 2008, PM speciation and sources in Mexico during the MILAGRO-2006 Campaign: Atmospheric Chemistry and Physics, 8, 111-128.

Rodríguez-Barrón, M.E., 2010, Caracterización geoestadística del subsuelo del exLago de Texcoco: México D.F., Universidad Nacional Autónoma de México, tesis de maestría, 208 p.

Ruiz, J.A., Juárez, M.C., Morales, M.P., López-Ochoa, L.M., Doménech, J., 2013a, Biomass gasification or combustion for generating electricity in Spain: Review of its current situation according to the opinion of specialists in the field: Journal of Renewable and Sustainable Energy, 5, 012801.

Ruiz, J.A., Juárez, M.C., Morales, M.P., Muñoz, P., Mendívil, M.A., 2013b, Biomass gasification for electricity generation: Review of current technology barriers: Renewable and Sustainable Energy Reviews, 18, 174-183.

Rzedowski, G.C. de, Rzedowski, J., 2005, Flora fanerogámica del Valle de México: Michoacán, México, INECOL, A.C. y CONABIO. 1406 p.

Sakai, S., Yoshida, H., Hirai, Y., Asari, M., Takigami, H., Takahashi, S., Tomoda, K., Peeler, M.V., Wejchert, J., Schmid-Unterseh, T., Douvan, A.R., Hathaway, R., Hylander, L.D., Fischer, C., Oh, G.J., Jinhui, L., Chi, N. K., 2011, International comparative study of 3R and waste management policy developments: Journal of Material Cycles and Waste Management, 13, 86-102.

Scopus, 2014, Analyze search results (TITLE-ABS-KEY (biomass gasification)). Scopus Base de datos, disponible en $<$ http://www. scopus.com/home.url>, consultado 6 de octubre de 2014.

Secretaría de Comunicaciones y Transportes (SCT), 2014, Un aeropuerto de calidad mundial para la Ciudad de México, artículo (en línea): Secretaría de Comunicaciones y Transportes, actualizado 26 septiembre de 2014, disponible en <http://www.sct.gob.mx/ despliega-noticias/article/un-aeropuerto-de-calidad-mundial-parala-ciudad-de-mexico/>, consultado 24 de septiembre de 2014.

Secretaría de Energía (SENER), 2012, Prospectiva de Energías Renovables 2012-2026: México, D.F., Secretaría de Energía, 156 p.

Secretaría del Medio Ambiente, GDF (SEDEMA), 2012, Inventario de emisiones de la Zona Metropolitana del Valle de México 2010. Contaminantes criterio: México, D.F., Secretaría del Medio Ambiente, GDF, 104 p.

Secretaría de Medio Ambiente y Recursos Naturales (SEMARNAT), 2013, Informe de la Situación del Medio Ambiente en México. Compendio de Estadísticas Ambientales. Indicadores Clave y de Desempeño Ambiental: México, D.F., Secretaría de Medio Ambiente y Recursos Naturales. 361 p.

Secretaría de Recursos Hidráulicos (SRH), 1971, Estudio Agrológico Especial del exLago de Texcoco, Estado de México: México, D.F., Secretaría de Recursos Hidráulicos, Dirección General de Irrigación y Control de Ríos, Serie de estudios No. 2, 145 p.

Siemens, 2010, Índice de Ciudades Verdes de América Latina. Una evaluación comparativa del impacto ecológico de las principales ciudades de América Latina (en línea): Alemania, Siemens AGEconomic Intelligence Unit, disponible en $<$ http://www.siemens. 
com/press/pool/de/events/corporate/2010-11-lam/Study-LatinAmerican-Green-City-Index_spain.pdf $>$, consultado 14 de abril de 2014.

Sistema de Aguas de la Ciudad de México (SACMEX), 2012, El gran reto del agua en la Ciudad de México. Pasado, presente y prospectivas de solución para una de las ciudades más complejas del mundo: México, D.F., Sistema de Aguas de la Ciudad de México, 191 p.

Sistema de Monitoreo Atmosférico (SIMAT), 2014a, Mosaico de la calidad del aire por partículas menores a 2.5 micrómetros en la Zona Metropolinata de la Ciudad de México (2004-2014), Año 4 , Número 43, disponible en $<$ http://www.aire.df.gob.mx/>, consultado 12 de octubre de 2014.

Sistema de Monitoreo Atmosférico (SIMAT), 2014b, Mosaico de la calidad del aire por partículas menores a 10 micrómetros en la Zona Metropolinata de la Ciudad de México (2000-2014), Año 6, Número 65 , disponible en $<\mathrm{http}: / /$ www.aire.df.gob.mx/>, consultado $12 \mathrm{de}$ octubre de 2014.

Solar Irradiance, 2014, Solar Irradiance calculator (en línea): Arizona, U.S., disponible en $<$ http://solarelectricityhandbook.com/solar-irradiance. html $>$, consultado 26 de mayo de 2014.

Sonneveld, P.J., Swinkels, G.L.A.M., van Tuijl, B.A.J., Janssen, H.J.J., Campen, J., Bot, G.P.A., 2011, Performance of a concentrated photovoltaic energy system with static linear Fresnel lenses: Solar Energy, 85(3), 432-442.

Stasch, D., 1996, Umweltverträglichkeit der Bodennutzung im Langenauer Ried, Hohenheimer Bodenkundliche Hefte, 30, Institut für Bodenkunde und Standortslehre, Universität Hohenheim, Stuttgart.
Tan, K.H., 1993, Principles of soil chemistry: New York, U.S., Marcel Dekker, Inc., $362 \mathrm{p}$.

Tetra Tech ES Inc., 2013, Experiencias internacionales en el composteo de residuos sólidos orgánicos, Programa para el Desarrollo Bajo en Emisiones de México (MLED): U.S., United States Agency for International Development (USAID), $83 \mathrm{p}$.

Torres-Guadarrama, T., 2011, Globalización y Ampliación del Aeropuerto Internacional de la Ciudad de México: México D.F., Instituto Politécnico Nacional, tesis de maestría, 203 p.

Verheijen, F., Jeffery, S., Bastos, A.C., van der Velde, M., Diafas, I., 2010, Biochar Application to Soils, A Critical Scientific Review of Effects on Soil Properties, Processes and Functions, European Commission, disponible en <http://publications.jrc.ec.europa.eu/repository/ handle/111111111/13558>, consultado 22 de septiembre de 2014.

Yano, A., Kadowaki, M., Furue, A., Tamaki, N., Tanaka, T., Hiraki, E., Kato, Y., Ishizu, F., Noda, S., 2010, Shading and electrical features of a photovoltaic array mounted inside the roof of an east-west oriented greenhouse, Biosystems Engineering, 106(4), 367-377.

Manuscrito recibido: Junio 20, 2014

Manuscrito corregido recibido: Octubre 22, 2014

Manuscrito aceptado: Noviembre 19, 2014 